To appear in Vehicle System Dynamics

Vol. 00, No. 00, Month 20XX, 1-34

\title{
Steering Disturbance Rejection using a Physics-based Neuromusculoskeletal Driver Model
}

\author{
Naser Mehrabi*, Reza Sharif Razavian and John McPhee \\ Systems Design Engineering, University of Waterloo, Waterloo, Canada \\ (Received 00 Month 20XX; accepted 00 Month 20XX)
}

\begin{abstract}
The aim of this work is to develop a comprehensive yet practical driver model to be used in studying driver-vehicle interactions. Drivers interact with their vehicle and the road through the steering wheel. This interaction forms a closed-loop coupled human-machine system, which influences the driver's steering feel and control performance. A hierarchical approach is proposed here to capture the complexity of the driver's neuromuscular dynamics and the central nervous system in the coordination of the driver's upper extremity activities, especially in the presence of external disturbance. The proposed motor control framework has three layers: the first (or the path planning) plans a desired vehicle trajectory and the required steering angles to perform the desired trajectory; the second (or the musculoskeletal controller) actuates the musculoskeletal arm to rotate the steering wheel accordingly; and the final layer ensures the precision control and disturbance rejection of the motor control units. The physics-based driver model presented here can also provide insights into vehicle control in relaxed and tensed driving conditions, which are simulated by adjusting the driver model parameters such as cognition delay and muscle co-contraction dynamics.
\end{abstract}

Keywords: musculoskeletal arm model, human driver model, disturbance rejection, stretch reflex, intrinsic muscle properties

\section{Introduction}

Realistic driver models and computer simulations can play an important role in designing and improving driver-assist technologies by reducing the cost and time associated with vehicle development. For example, a high-fidelity driver model, which considers the physiological limitations and attributes of a human, can reasonably approximate a

*Corresponding author. Email: nmehrabi@uwaterloo.ca 
real driver's steering behavior; as such, it can be used to study the performance of new driver-assist technologies [1].

Driver models can be categorized into vehicle-based and human-based [2, 3]. A vehiclebased driver model will calculate the steering angle/torque required to perform a given maneuver [4-7], but it does not consider how this torque is produced, or if the produced torque is physiologically possible. In contrast, a human-based (physiological) driver model is focused on the understanding of the driver's limitation and preferences. Together, the driver and the vehicle form a coupled human-machine system, which should be treated as a whole in the design process of driver-assist technologies.

Studies of coupled human-machine systems date back to the early work on aircraft pilots done by Weir and McRuer [8] in 1970. Those studies helped to reveal various properties and characteristics of humans as adaptive controllers of dynamic systems. Since then, more specific studies considered the automobile driver's neuromuscular system to understand the behavior of human vehicle drivers [9-13]. Physical limitations and attributes of the human driver, such as the required processing and transition time for sensory information to reach the brain, the cognitive requirement to anticipate or perceive the information, and muscle contraction dynamics, help to characterize the human control behavior. For example, Pick and Cole, in a series of papers [13-16], reported the passive dynamics of driver arms. They showed that the angle and torque measurements of a steering wheel held by a driver can be represented by a lumped system at the steering wheel, and can easily be fitted to a second-order transfer function of inertia, damping, and stiffness. Non-linear driver models with three-dimensional (3D) musculoskeletal arm models were developed in $[9,17,18]$ to study steering tasks for a wide range of steering motions. However, these studies did not include the effects of muscle dynamics, muscle co-contraction, and stretch reflex. The aim of our research is to include these elements in the motor control framework of the human driver model to study steering tasks in the presence of external disturbances. Motor control, especially the disturbance rejection mechanism of the human musculoskeletal system, is highly non-linear; therefore, a fairly sophisticated system is required to capture its dynamics. In this research, a novel hierarchical framework is proposed to model the complexities of human motor control of steering.

The Central Nervous System (CNS) is responsible for processing all information from and to the peripheral nervous system. It can modulate the motor commands based on 
the available sensory information, and its internal knowledge of the body and the environment to coordinate the voluntary movements of the body. The CNS can maintain the limb position in space despite external perturbations such as external loads, e.g., the act of stabilizing the steering wheel when tires hit a curb/road irregularity or when the wind gusts. These commands lead to voluntary and involuntary driver actions, which respectively result in long and short latency reflexes to maintain a desired position.

It is a well-established fact $[19,20]$ that the CNS uses two mechanisms to stabilize a limb in the presence of external disturbances. The first one is based on intrinsic properties (mechanical resistance) of the individual muscles. The CNS can increase the resistance of the joint against the external disturbances by co-contracting all the muscles wrapping around a joint. The Hill-type muscle model shown in $[21,22]$ can be used to simulate the total mechanical resistance of the limb. However, different combinations of the muscle elements have been used in the literature to simplify the mathematical representation of muscle dynamics to study joint stability. A Hill-type model can be variously configured; for instance, a contractile element in series with a tendon $[23,24]$ or a contractile element in parallel with connective tissues [25] have been used to simulate the muscle dynamics. It has been demonstrated in [26] that stabilizing a limb requires at least a pair of agonist and antagonistic muscles. The stabilization is achieved by increasing the mechanical resistance of the joint by raising the amount of antagonistic co-contraction [9, 20, 26].

The second mechanism is based on the proprioceptive feedback. Proprioceptors consist of spindles and Golgi tendon organs that sense the relative movements and orientation of limbs. For example, the primary $(I a)$ afferent fibers of muscle spindles sense the changes in the muscle length and contraction velocity. These feedbacks program the $\gamma$-motor neurons with the expectation of how the muscle should shorten during the movement. Short-loop pathway reflexes, such as mono-synaptic actions of the Ia afferent on $\alpha$-motor neurons, allow the nervous system to quickly correct errors in the muscle length (e.g., the knee-jerk response). This mechanism offers a fast and energy-efficient solution to quickly reject a disturbance.

$\alpha$-motor neurons innervate muscle fibers, and therefore are directly responsible for muscle contraction, while $\gamma$-motor neurons indirectly contract muscle fibers by adjusting the sensitivity of the muscle spindles to control the firing rate of $\alpha$-motor neurons. This phenomenon is called the co-activation of $\alpha$ and $\gamma$ motor neurons. The co-activation of $\alpha$ and $\gamma$ signals in driver models is studied in $[16,27,28]$. In these articles, however, 
the $\alpha$ and $\gamma$ signals are represented as lumped variables, working at the level of steering wheel angle and torque, and not at the muscle level. Thus, their biological significance (relationship between muscle length and activation) is not properly represented.

To the best of the authors' knowledge, muscle-level stretch reflex and voluntary musculoskeletal response to disturbance have not been used to study a driver's behavior in the presence of an external disturbance. The muscle co-contraction ratio is included in the driver model so that the effects of driving with relaxed and tensed muscles can be studied.

This paper is organized as follows. In the second section, the 3D musculoskeletal driver model is presented. The hierarchical motor control framework is described in detail in section 3. Section 4 presents the numerical results and discuses the steering task. Finally, the conclusions and plans for future research directions are presented in section 5 .

\section{Integrated Vehicle-Driver Model Description}

Before getting into the details of the driver and vehicle models, we would like to explain the difference between two types of models that we have used in our simulations: the highfidelity models and the simplified models. The high-fidelity ones (sections 2.1 and 2.2) try to faithfully replicate the real system, and are used to obtain the simulation outputs. The simplified models, on the other hand, do not necessarily contain all the details of the system, and are only used to help to predict the real system. These simplified models are confined within the motor control systems (sections 3.1 and 3.2), and are solved multiple times at each time step. Therefore, the simplicity of the models is vital in keeping the computational burden manageable. The validity of both types of models is equally important, as the errors in either will affect the reliability of the results. Additionally, due to the interaction of the models, error in the any of models will hinder the interpretation of the results.

\subsection{D Musculoskeletal Arm Model}

Simple neuromuscular driver models have been reported in the literature [13, 29], but their range of motion is essentially limited due to their linear nature. Therefore, to reliably study steering tasks, a model with a larger range of operations needs to be employed. In this article, a modified version of a three-dimensional musculoskeletal arm 


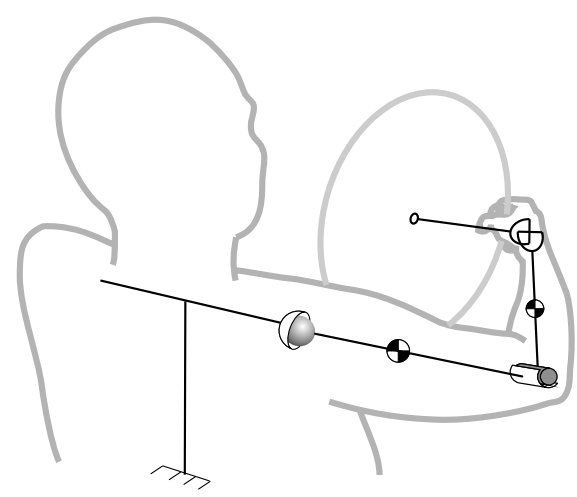

(a)

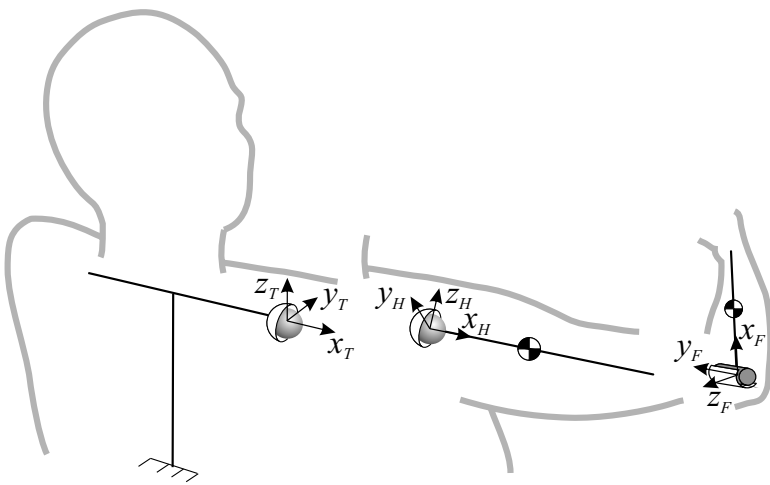

(b)

Figure 1. (a) Schematic view of the 3D arm model. (b) Coordinate systems for torso (T), humerus (H), and forearm ( $\mathrm{F})$ (ulna and radius).

model developed earlier by the authors $[9,17]$ is used.

Figure 1 shows the schematic view of the 3D arm model. Its torso (scapula, assumed fixed relative to the vehicle) is attached to the upper arm (humerus) via a spherical joint. The forearm (ulna and radius) is, in turn, connected to the upper arm via a revolute joint to allow for flexion/extension of the elbow. Finally, a universal joint is used to connect the forearm to the hand, allowing the flexion/extension and abduction/adduction of the wrist joint. It is also assumed that the hand firmly grips the steering wheel; thus, the hand/steering wheel connection is modeled as a fixed (weld) joint.

Since the deltoid muscle comprises approximately one-fifth of shoulder muscles [30, 31], the three main portions (anterior, middle, and posterior) of deltoid muscle are modeled separately here to improve the fidelity of the model. Therefore, unlike the upper arm model shown in [17] in which thirteen muscles were used, the current model employs fifteen muscles to articulate the upper limb. In the new model, eight muscles cross the shoulder, and the remaining seven actuate the elbow. Similar to [17], the muscles associated with forearm supination/pronation (Supinator, Pronator Teres and Subscapularis) and wrist muscles are not included in the current model in order to reduce the computational burden of the muscle redundancy problem.

In this article, a Hill-type model is used to simulate the muscle contraction dynamics $[21,22]$. Since the Hill muscle model is based on empirical relations, it is computationally less demanding than other microscopic approaches [32] and is computationally manageable for a system with several muscles. Furthermore, the Hill muscle model parameters can be easily adjusted to represent age-related and gender-related changes in muscle mechanical properties. The Hill muscle model consists of a Contractile Element (CE) 


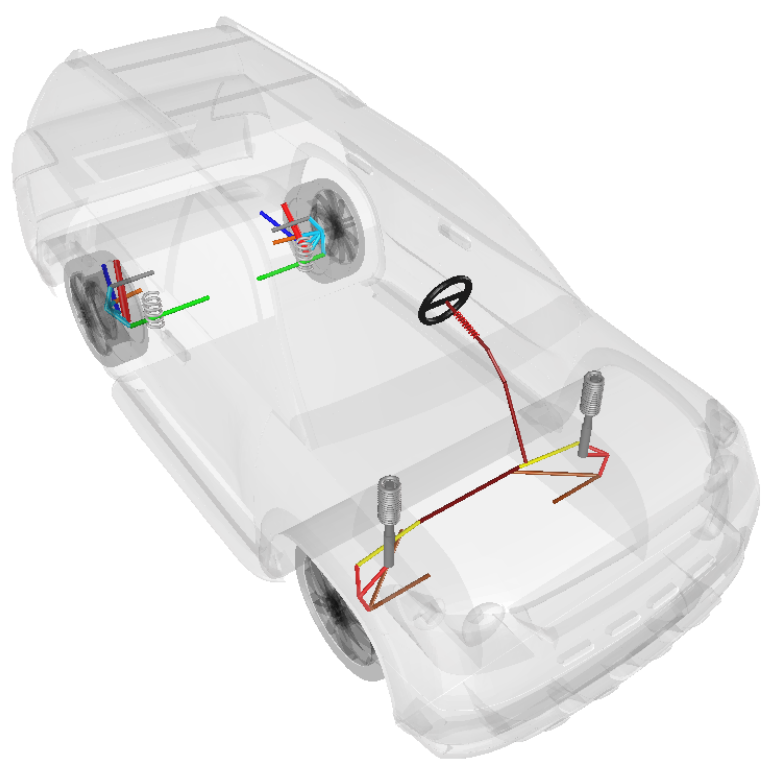

(a)

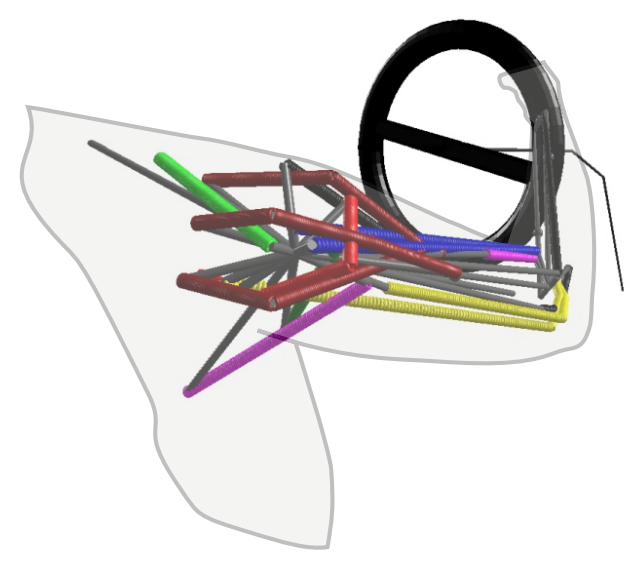

(b)

Figure 2. The 3D arm and the vehicle models in MapleSim (a) A view of the vehicle model (b) The muscleactuated 3D arm model

and a Parallel Elastic element (PE) in series with a Series Elastic element (SE). In this research, the tendon dynamics (SE) are neglected, as the motion is relatively slow and the amount of energy transfer in tendons is small. However, stiffness produced by the SE element of muscles wrapping the shoulder and elbow joints, as a result of muscle co-contraction, is approximated by stiffness and viscoelastic damping at the joints.

Details of the muscle model are given in Appendix B. This musculoskeletal model of a driver's arm is validated using surface electromyography (EMG) of a human driver's right arm performing two steering maneuvers in a driving simulator [33-35]. The muscle path parameters from [17] are slightly re-tuned to provide better correlation between the experiments and model predictions. The muscle path parameters used in this article are summarized in Table C1 of Appendix C. The plausible range of steering motion for the driver model with both hands on the steering wheel is $-90^{\circ}$ to $+90^{\circ}$; beyond this range, the kinematic constraints cannot be satisfied anymore and the arm mechanism locks up.

\subsection{Vehicle Dynamics}

To reliably simulate the driver-vehicle interaction, the vehicle dynamics has to be faithfully modeled as well. In this research, a vehicle model with a front MacPherson suspension and a rear multilink/semi-trailing arm suspension typical in SUVs (Sport Utility 


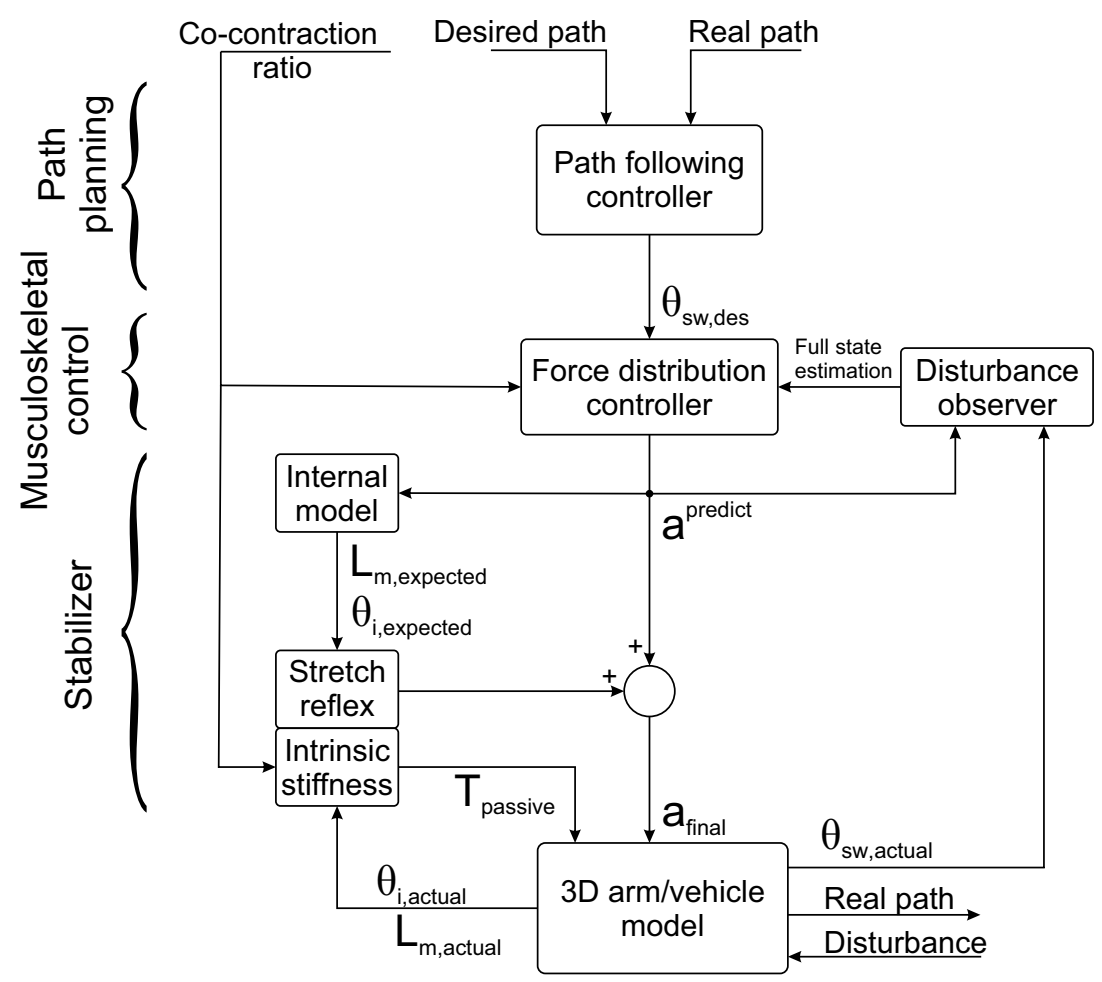

Figure 3. The motor control framework to study steering task

Vehicle) is developed in MapleSim as shown in Fig 2(a). The symbolic modeling engine of MapleSim is able to generate optimized C code describing the system's equations of motion. These symbolically generated equations can reduce the simulation time significantly and allow extensive manipulation of the system's equations.

The developed model consists of 50 generalized coordinates coupled by 34 algebraic equations. This model in total has 16 degrees of freedom (DOF). Six DOF are associated with the rigid body motion, four are related to the wheels' spin, four are related to the suspensions' vertical motion and two are associated with the steering motion. Since the vehicle model development is not the focus of this paper, the vehicle model details are not included in this article, and more information about the high-fidelity vehicle model can be found in [35-37].

\section{Motor control framework of driver}

A feature-rich motor control framework for the musculoskeletal driver model considering the sensory and actuator dynamics of human limb has been developed here to study the driver/vehicle interaction. In humans, the CNS comprises information processing 
in the brain, the cerebellum and the neural circuits of spinal cord, to modulate motor commands and control the voluntary and the involuntary actions of the driver.

To reliably study motor action tasks, we need to establish a predictive framework with sufficient bio-fidelity. With the motor control framework presented in this article, we can define an environment (which may include disturbances and uncertain dynamics) and a desired steering action, run the simulation, and observe the consequences. Such a model can help to study steering tasks and design new driver-assist technologies.

As shown in Fig. 3, the proposed motor control framework consists of three hierarchies: the path planning, the musculoskeletal control, and finally the stabilizer. The path planning controller (level one of control hierarchy) works in the low-dimensional end-effector space (in this context the steering wheel angle); it defines a desired steering wheel trajectory, $\theta_{s w, d e s}$, based on its internal representation of the system. The musculoskeletal controller (level two in the hierarchy), works in the high-dimensional muscle space, and tries to predict the muscle activation, $a^{\text {predict }}$, required to follow the high-level controller's set points. In the stabilizer part, the stretch reflex (the lowest level in the hierarchy) also works in high-dimensional muscle space, and the intrinsic stiffness module works in the joint space. The lowest level of hierarchy is responsible for compensating for inaccuracies and disturbances in the system. The path-following controller and musculoskeletal controller shown in [17] are modified and used here in the first and second layers of the motor control framework, respectively.

\subsection{Level one - path planning controller}

The first layer of the framework, the path planning, estimates the required steering wheel angle to perform a specific task, and is represented by a model predictive controller (MPC) $[17,38]$.

As argued by Kim and Cole [39], the cerebellum may contain representations of the nonlinear body/vehicle dynamics, in the form of a set of linear models (internal models). The versatility of this set shows the driver steering skill, which results in better performance of the driver in the near-limit conditions. In this article, a single linear model, consists of a linear bicycle model with a linear tire model as shown in Appendix A, is used to represent the driver's steering skill. Since all the predictions in the path planning are based on this internal model, this model should be able to replicate dynamics of the yawing and lateral motion of the vehicle. Figure 4(b) shows the comparison of the 


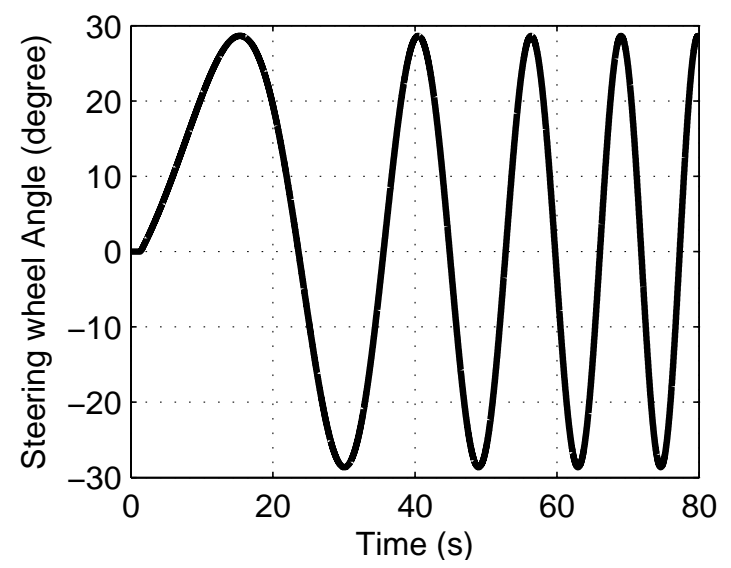

(a)

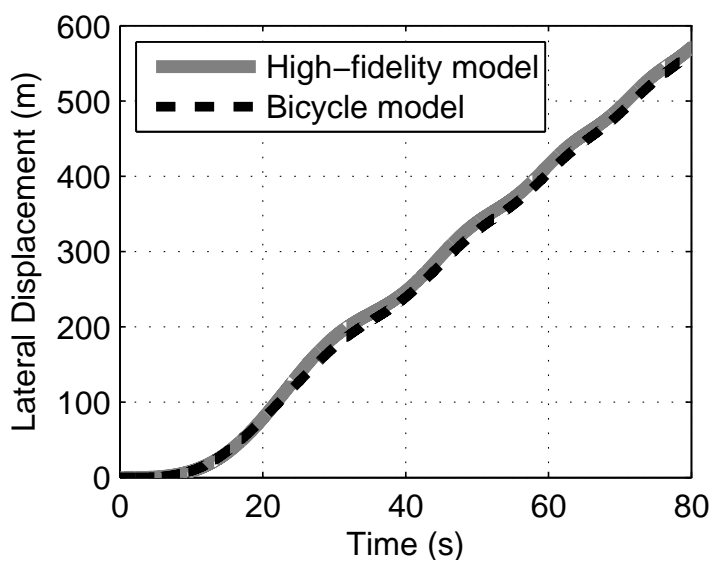

(b)

Figure 4. System response for the high fidelity vehicle and the bicycle models (a) The steering wheel angle as the input to the systems (b) The lateral position as the output of the systems

lateral response to a sweep sinusoidal steering wheel angle (shown in Fig. 4(a)) for the high-fidelity and this internal model at a constant speed of $10 \mathrm{~m} / \mathrm{s}$.

The MPC path planning controller use the internal model to predict the best steering wheel trajectory by solving an optimization problem. In this article, the Model Predictive Control toolbox [40] of Matlab is used to implement the path planning controller. In this implementation of MPC, the time is discretized into $10 \mathrm{~ms}$ intervals, in which the control inputs (the steering wheel angle) are assumed to be constant. MPC finds the optimal sequence of control input (with the control horizon length) over the prediction horizon length resulting in the optimal tracking of the desired lateral displacement and yaw rate of the vehicle. Then, the MPC selects the initial element of the sequence and applies it to the high-fidelity model.

To study the effect of prediction and control horizon lengths on the path planning performance of the driver model, two simulation studies using the internal model are performed. In these simulations, a step signal with amplitude of $3 m$ is set as the desired lateral trajectory of the vehicle, as shown in Fig. 6. Since the desired trajectory is a sharp step signal, the path planning controller should begin steering earlier than when the step arises. This behavior of MPC clearly mimics the predictive path planning ability of human drivers. In the first set of simulations, the prediction and control horizons of the MPC controller are kept identical, and raised from looking $1 s\left(n_{p}=n_{c}=100 \times 0.01=1 s\right)$ ahead of the vehicle to $3 s\left(n_{p}=n_{c}=300 \times 0.01=3 s\right)$, consecutively. Figure 5 shows the steering wheel angle calculated by the MPC to perform the lane change maneuver. 


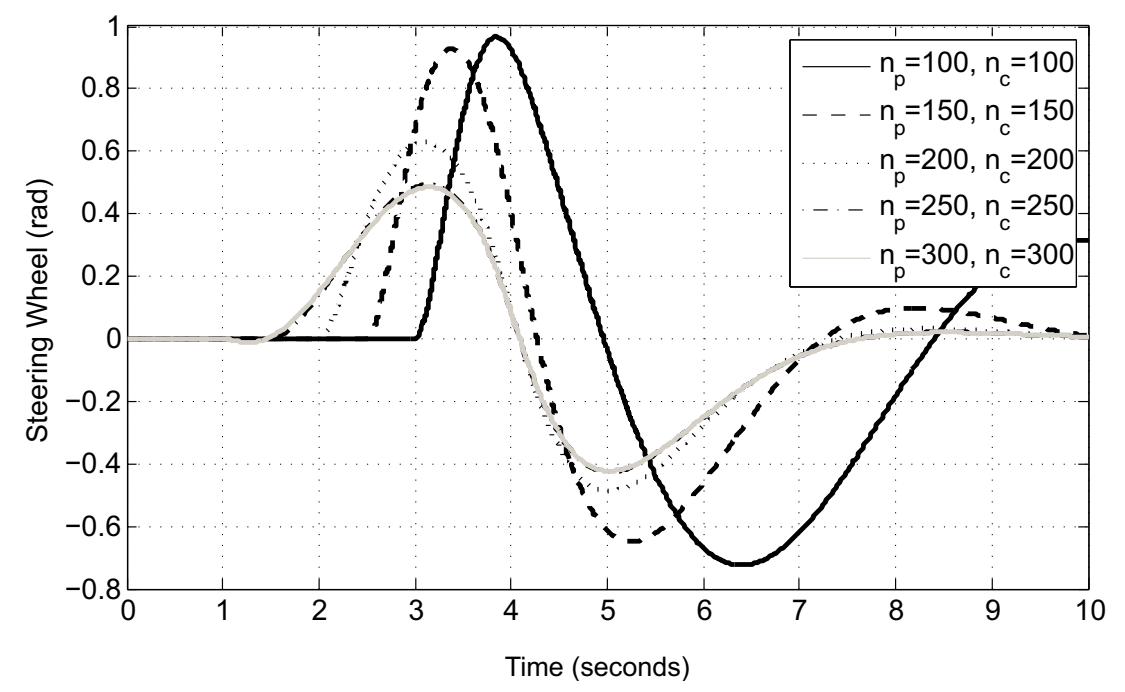

Figure 5. The effect of prediction and control horizon of MPC path planning controller on the steering wheel angle variation
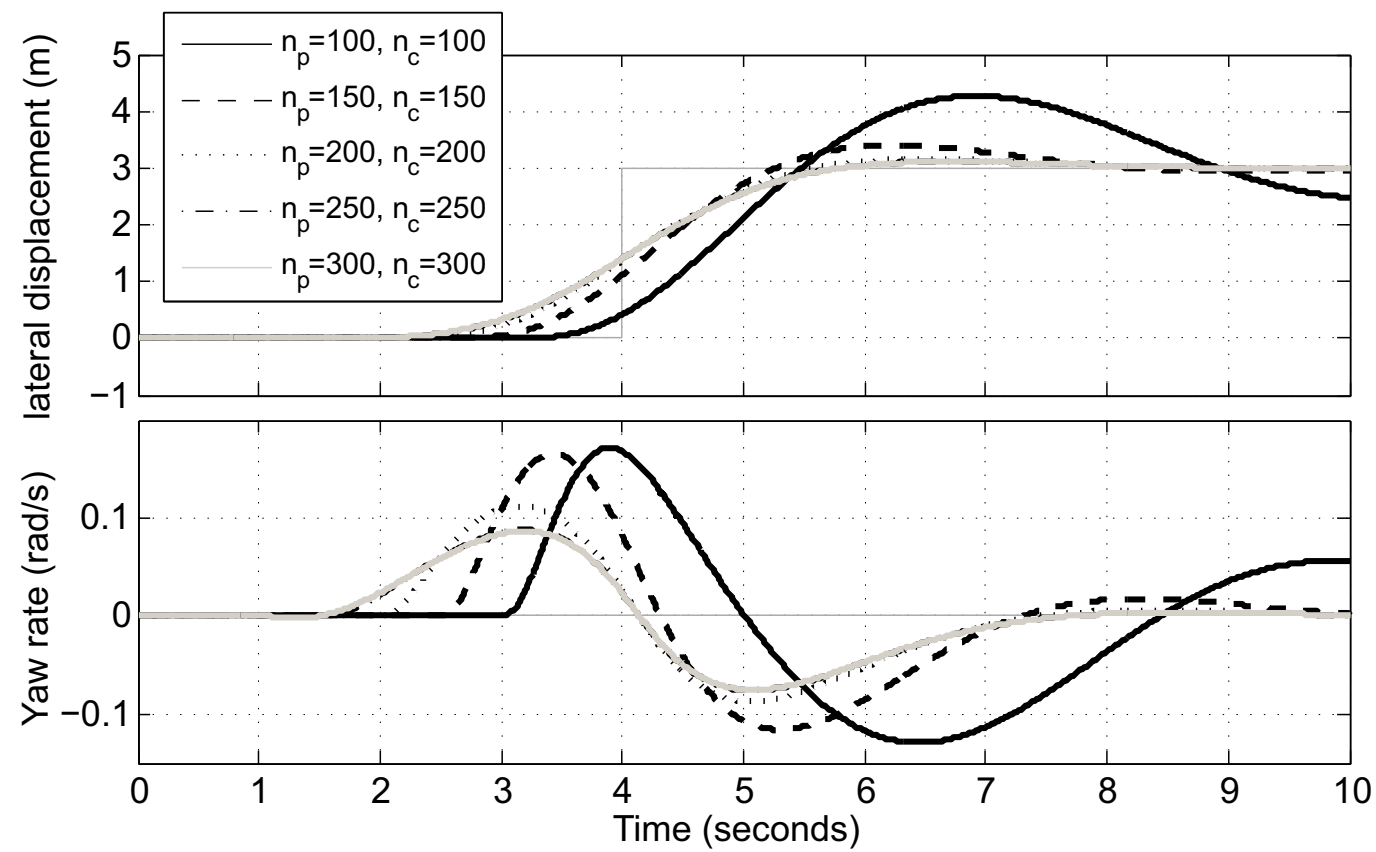

Figure 6. The effect of control and prediction horizon lengths of MPC path planning controller on the vehicle lateral displacement and yaw rate

It can be seen that with the prediction horizon of $1 s$, the driver model cannot manage to perform the lane change in the specified time. By increasing the horizons, the perfect lane change can be achieved. As shown in Fig. 6, with prediction horizons over the $2.5 \mathrm{~s}$, the vehicle actual path does not change significantly. Therefore, $2.5 s$ is chosen as the prediction horizon length in the remaining of this article.

In the second set of simulations, to study the influence of control horizon on the per- 


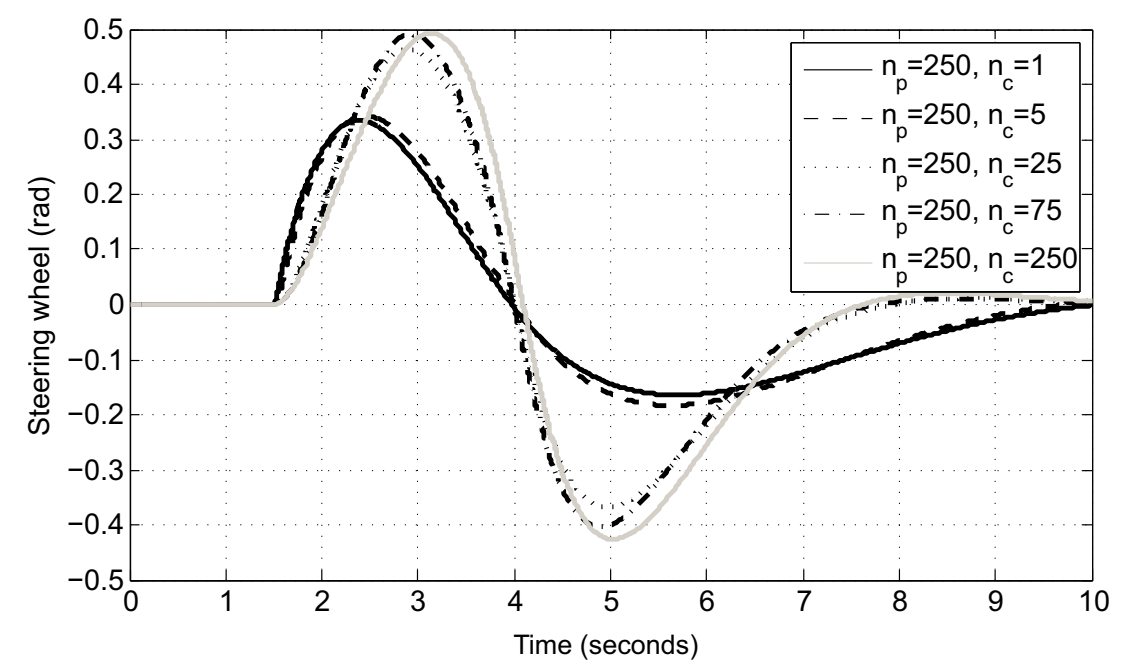

Figure 7. The effect of control horizon of MPC path planning controller on the steering wheel angle variation
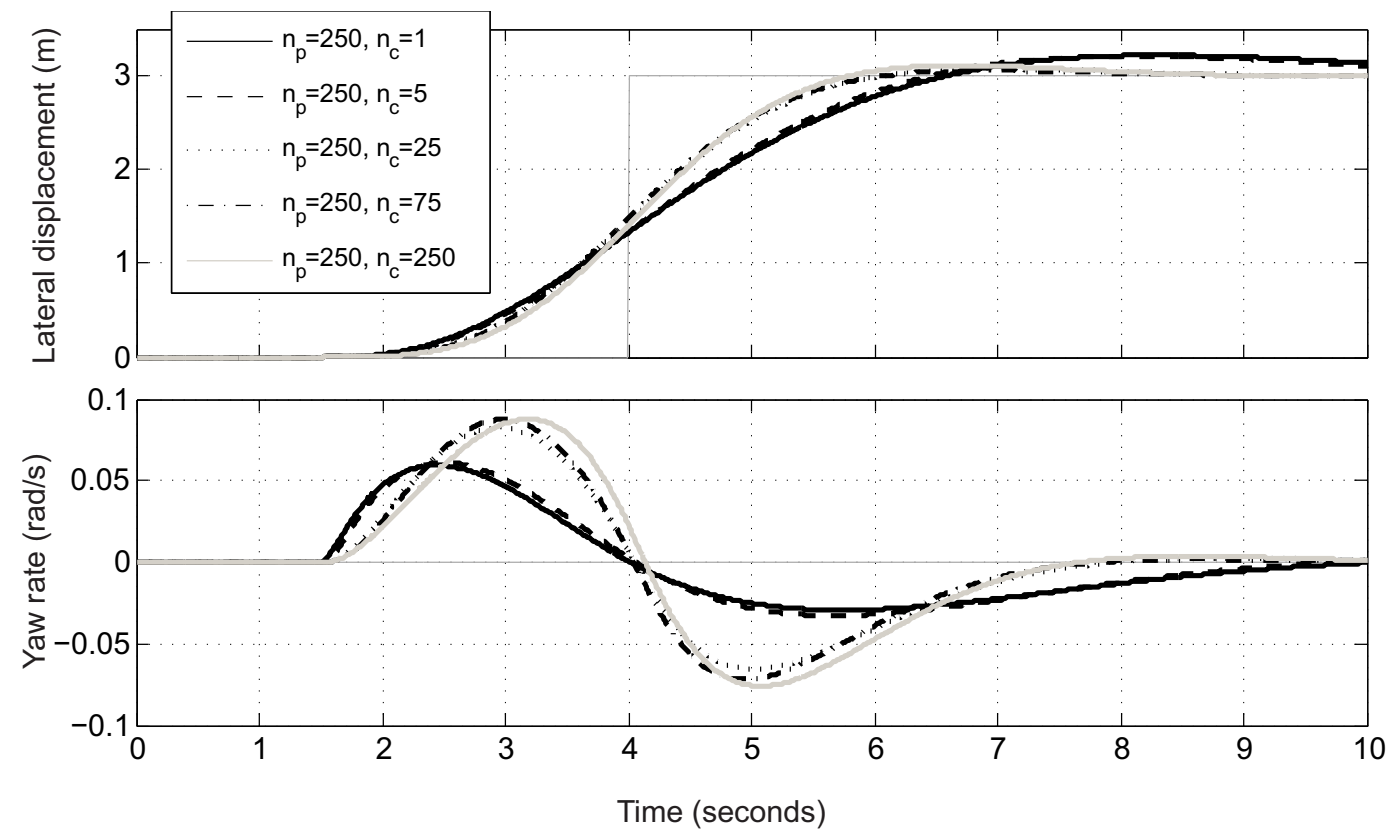

Figure 8. The effect of control horizon length of MPC path planning controller on the vehicle lateral displacement and yaw rate

formance of controller, the prediction horizon kept constant and equal to $2.5 s$ while the controller horizon increases gradually. As shown in Fig. 8, with a single interval control horizon $\left(n_{c}=1 \times 0.01=0.01 s\right)$, the performance of controller is degraded; nonetheless, the closed-loop system is stable. By increasing the control horizon, the performance of controller converges to the case with full length control horizon; however, the increase more than $0.25 s$ only slightly improves the performance of the controller, while notably increases the computational expense. Figure 7 shows the required steering wheel angle to 
perform the lane change maneuver corresponding to the varied prediction control length. Therefore, in our path planning MPC, the prediction horizon length is 250 intervals $(250 \times 0.01=2.5 \mathrm{~s})$ and the control horizon length is 25 intervals $(25 \times 0.01=0.25 \mathrm{~s})$.

\subsection{Level two - force distribution controller and disturbance observer}

The second layer of the motor control framework, the musculoskeletal control, includes the Force Distribution (FD) controller and the disturbance observer. This layer represents the process of information collection from the sensory organs and the control of conscious voluntary actions of the upper limb.

In this article, the FD controller assigns the required muscle activations to perform a specific arm motion. The development of the FD controller has been inspired from a well-known motor control hypothesis [41, 42]: it postulates that the CNS minimizes a physiological cost function while performing a motion. However, the configuration and states of the body should be known to the CNS/FD controller to find the optimal muscle activations. Therefore, an observer is required to estimate the state variables during the motion. In the following sections, the FD controller and the disturbance observer are presented.

\subsubsection{Force distribution controller}

Similar to the path planning controller, the system behavior (steering wheel rotation as a result of the muscle activity) is predicted by an internal representation of the system, called the vehicle-driver internal model. Here, the resistive steering torque at the steering wheel in conjunction with the 3D model of arm (section 2.1) holding the steering wheel is selected as the vehicle-driver internal model.

The resistive torque is approximated with a passive torque (see Eq. (1)) at the steering column to accelerate the simulations. Figure 9(b) demonstrates the comparison of prediction of the passive torque and the high-fidelity model of vehicle steered with right hand only (in terms of the resistive torque). In this figure, the resistive steering torque is shown when the steering wheel angle follows a sinusoidal trajectory at speed of $10 \mathrm{~m} / \mathrm{s}$ (see Fig. 9(a)). The passive resistive torque at the steering wheel, $T_{s w}$, is given by

$$
T_{s w}=K_{s w} \theta_{s w}+C_{s w} \dot{\theta}_{s w}
$$




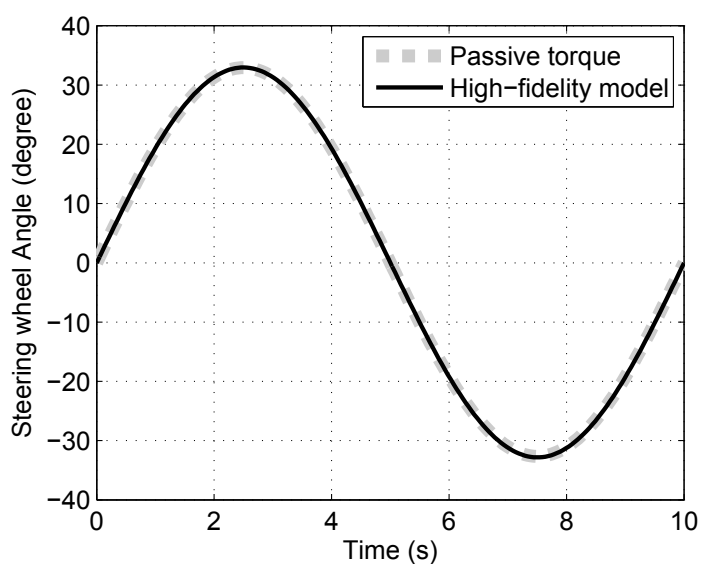

(a)

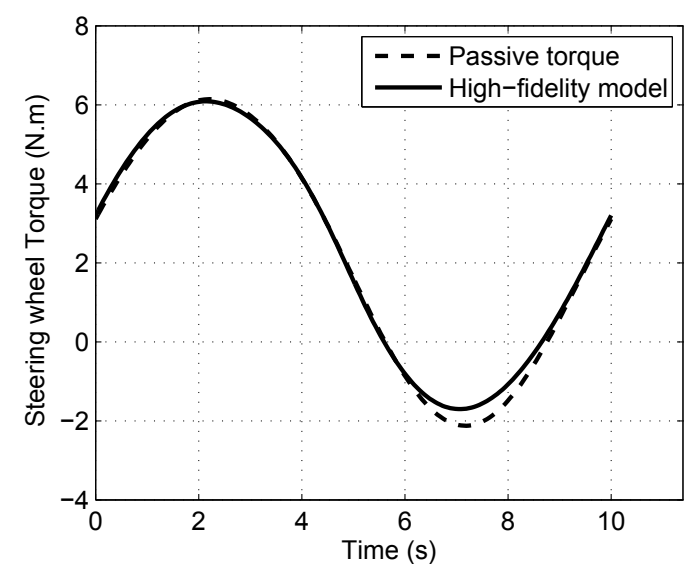

(b)

Figure 9. Simplified model resistive steering torque for a sinusoidal steering angle trajectory, compared against the high fidelity model: (a) Steering wheel angle (b) resistive steering torque

where the stiffness, $K_{s w}$, and the damping coefficients, $C_{s w}$, are found so that the internal model response matches the real vehicle dynamics as closely as possible within the linear response range. $K_{s w}$ and $C_{s w}$ are functions of vehicle driving condition such as vehicle speed, road coefficient of friction, etc.

The output of the path planning controller is the low-dimensional value for the steering wheel angle $\theta_{s w, d e s}$. To perform the steering task, the desired steering wheel angle must be transformed into the high-dimensional muscle activation space. This transformation can be performed by an optimization routine that minimizes the steering wheel angle tracking error. The major challenge associated with such a transformation is the inherent redundancy of the problem (there are more actuators than required), which results in infinite combinations of muscle activations that can track the desired steering wheel angle. However, the redundancy issue can be addressed by including an additional criterion when solving for the muscle activations $a^{\text {predict }}$. Such criteria are usually chosen to minimize a form of the physiological cost (such as muscle force or muscle fatigue) while maintaining the desired response. In this research, the muscle fatigue representation shown in Eq. (2) is used as the physiological cost.

As suggested in [43, 44], drivers tend to co-contract their muscles when their knowledge of the steering torque feedback behavior is inaccurate. In addition, the co-contraction of muscles wrapping a joint increases the stiffness and viscoelasticity of that joint, and therefore improves the joint stabilization and control accuracy [45]. In this research, to simulate steering with low and high muscle co-contractions, a muscle co-contraction ratio 
$\left(\alpha_{i}\right)$ has been added to the physiological cost function [46], as shown in Eq. (2).

$$
G(\mathbf{a})=\sum_{i=1}^{n}\left(a_{i}-\alpha_{i}\right)^{p}
$$

where the symbols $a_{i}$ and $\alpha_{i}$ represent the individual muscle activations, and the muscle co-contraction ratios, respectively. The exponent $p$ is chosen to be 2 in the simulations, and the summation accounts for all muscles $(n=15)$.

Additionally, since our motor control framework is a forward dynamic simulation (i.e. the applied forces generate the motion), tracking the desired motion is challenging. To ensure that the applied forces result in the desired motion, the tracking error is also included as a separate term into the FD controller cost function.

$$
a_{i}^{\text {predict }}[k]=\arg \min \left\{w_{1}\left(\theta_{s w}[k]-\theta_{s w, d e s}[k]\right)^{2}+w_{2} G(\mathbf{a}[k])\right\}
$$

where $\theta_{s w}[k]$ is the resultant steering wheel at time step $k$, which is compared against the desired value $\left(\theta_{s w, d e s}[k]\right.$, defined by the path planning controller $)$, and $w_{1}$ and $w_{2}$ are the weighting factors in the cost function.

The minimization can be performed at each time step (Forward Static Optimization [17]) or over the entire time span (Dynamic Optimization [47, 48]). In this research, the former approach is selected. At each time step, the force distribution controller solves a constrained optimization problem, using the sequential quadratic programming (SQP) optimization routine, to minimize both the physiological effort and the tracking error (Eq. (3)), while keeping the activations in the range $a_{i} \in[0,1]$.

Figure 10(b) shows the performance of the second layer of motor control framework in performing a sinusoidal steering maneuver. In this simulation, the driver holds the steering wheel with two hands at the 3 and 9 o'clock positions, as shown in Fig. 10(a). Figure 11 illustrates the right and left shoulder muscle activations to perform the steering task. As shown in Figs. 11(a) and 11(b), the shoulder muscles can be classified into two groups: those providing counterclockwise steering torque (group I), and muscles providing a clockwise steering torque (group II). Group I consists of the long head of triceps, latissimus dorsi and posterior portion of deltoid, and group II consists of anterior 


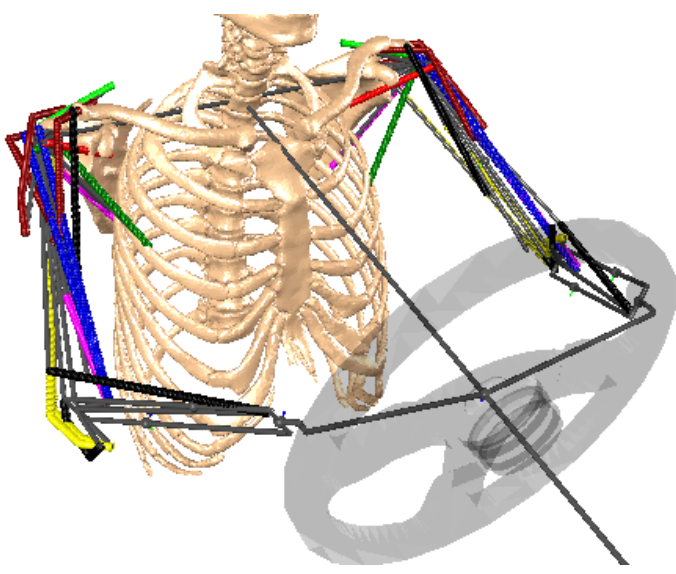

(a)

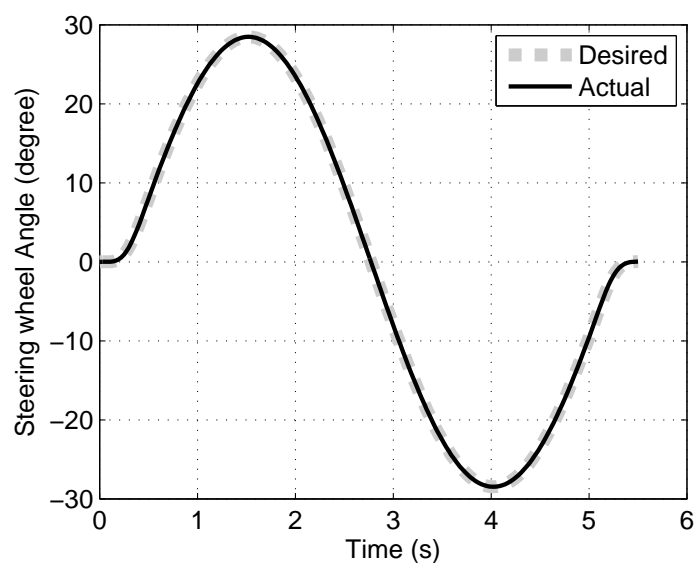

(b)

Figure 10. (a) Schematic view of the driver model in MapleSim with two hands on the steering wheel, (b) The sinusoidal steering wheel angle maneuver

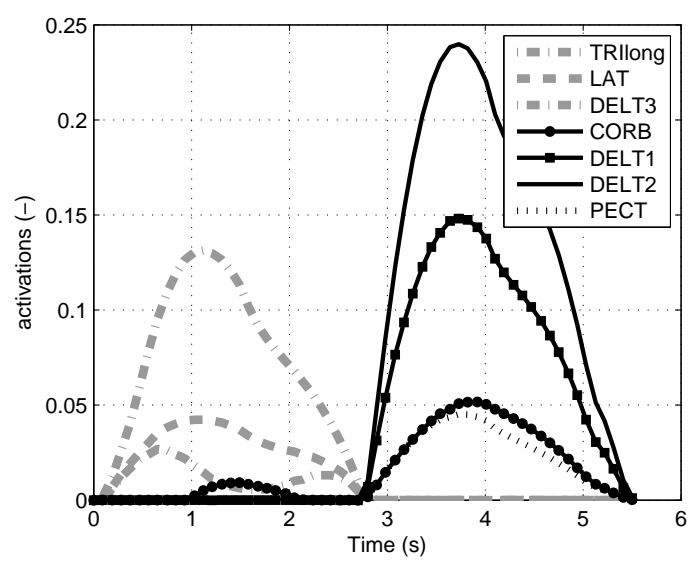

(a)

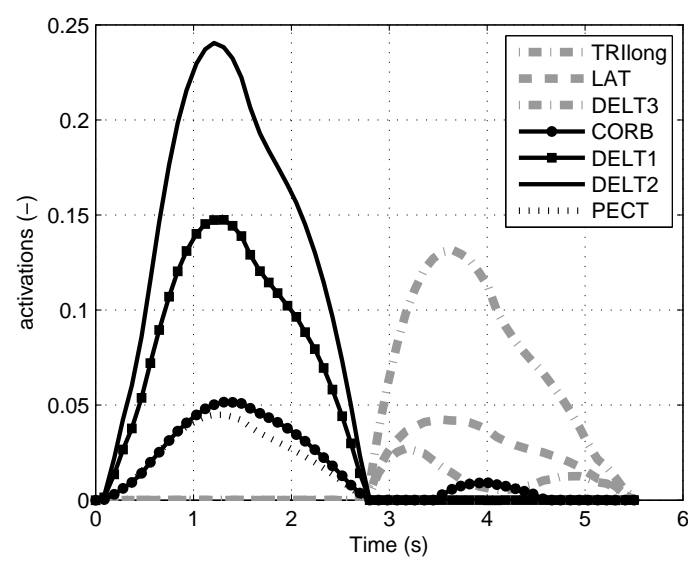

(b)

Figure 11. Sinusoidal steering wheel task (a) Right arm shoulder muscle forces, (b) Left arm shoulder muscle forces

and middle portions of deltoid, pectoralis major and coracobrachialis. When the driver turns the steering wheel clockwise, the group I muscles of the right arm and the group II muscles of the left arm are activated, and in the counterclockwise rotation, the opposite muscles of each arm are involved.

\subsubsection{Disturbance observer}

The FD controller needs the steering and body states to predict the optimal muscle activations. In this research, a disturbance observer is used to estimate the arm and steering 
state variables as well as the external disturbance torque at the steering wheel. From the biological point of view, the disturbance observer is used to replace the kinaesthetic sensory system and the predictor/corrector process of the CNS internal model structure to estimate the limb position.

If there is no disturbance in the system, the estimated states are similar to the actual states of the system, since they have started from the same initial condition and have similar dynamics. In this situation, the identified disturbance torque accounts for the small differences between the driver-vehicle internal model (passive torque) and the actual resistive steering torque. However, when the system states are altered by an external disturbance, there is an error in the estimated states. To correct this error, a feedback loop on the steering wheel position is added to the observer to identify the disturbance at the steering wheel and predict the altered states, as shown in Fig. 12(a).

The feedback estimator is a proportional-integral controller with a time delay. The time delay is associated with vestibulo-ocular and signal processing of the CNS, which results a latency in the the disturbance observer/force distribution performance. The transfer function of the feedback loop used to identify the disturbance torque is,

$$
H^{e}(S)=\frac{\hat{T}_{\text {disturbance }}(S)}{\Delta \theta_{\mathrm{sw}}(S)}=\left(K_{P}^{e}+\frac{K_{I}^{e}}{S}\right) e^{-\tau_{d}^{e} S}
$$

where $\hat{T}_{\text {disturbance }}$ and $\Delta \theta_{\mathrm{sw}}$ are the estimated disturbance torque and the steering wheel angle error, and $K_{P}^{e}$ and $K_{I}^{e}$ are the proportional and integral coefficients, respectively and $\tau_{d}^{e}$ is the time delay associated with the biological signal processing.

Figure 12(b) shows the performance of the disturbance observer in identifying a disturbance torque at the steering wheel. In this simulation, the car is driving in a straight line when a sudden unknown disturbance is applied to the car, which results in a pulse shape steering torque at the steering wheel. The observer starts to identify the disturbance after $300 \mathrm{~ms}$, reaches the actual disturbance after one second, and then returns to zero again.

\subsection{Level three - stretch reflex/intrinsic stiffness}

The third layer of the motor control framework, the stabilizer, stabilizes the limb and reduces the error introduced by external disturbances. This layer includes the stretch reflex and the intrinsic stiffness modulation mechanisms. The stretch reflex uses the 


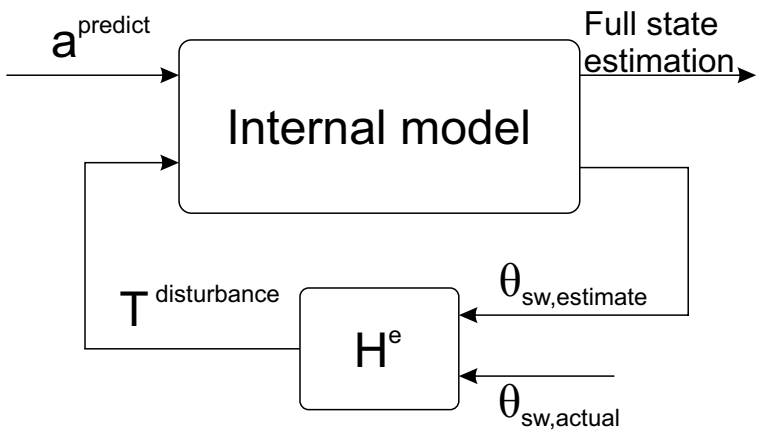

(a)

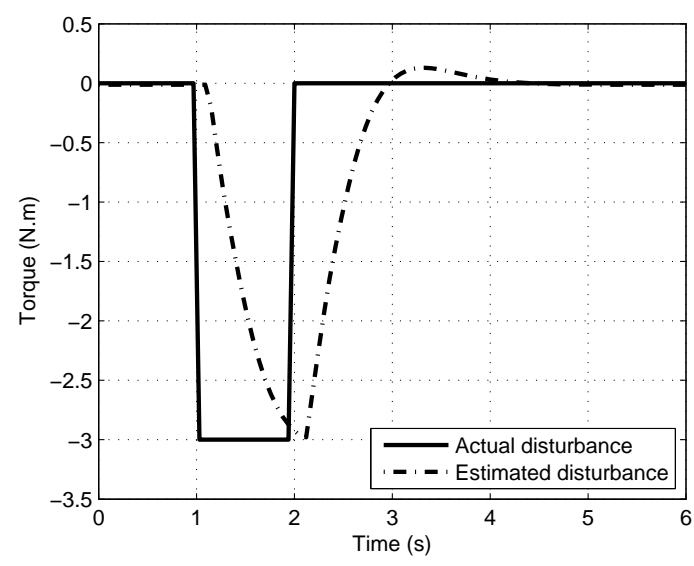

(b)

Figure 12. Disturbance observer mechanism and performance (a) The mathematical realization of the disturbance observer in the motor control framework (b) The disturbance torque at the steering wheel estimated by the disturbance observer

muscle proprioceptive feedback of the muscle spindles to regulate the muscle length, and the intrinsic stiffness modulates the visco-elastic properties of the muscles by cocontracting muscles wrapping a joint to stand against external disturbances.

The involuntary response due to stretch reflex occurs more quickly than the voluntary actions. In spite of the difference in their nature and response time, both the involuntary stretch reflex and the voluntary co-contraction contribute to movement stabilization.

\subsubsection{Stretch reflex}

In human anatomy, the $\gamma$-motoneuron activity and the stretch reflex are thought to be important mechanisms in improving motion accuracy and attenuating unwanted motions $[49,50]$. During a voluntary action, the nervous system has expected settings for muscle lengths; such expected lengths are set by adjusting the muscle spindle sensitivity, which in turn is done by modulating the $\gamma$-motoneuron activities. If due to a change in environment, the muscle length diverges from the expected value (i.e. over-stretched), then the $I a$ afferent activity will increase. Because of the excitatory synapse between the $I a$ afferent and $\alpha$-motoneurons, such increase in $I a$ activity will boost the $\alpha$-motor neuron activity which results in more muscle force that resists the muscle stretch. The stretch reflex can therefore provide disturbance rejection properties. In this research, the shortloop monosynaptic spinal stretch reflex is considered as the most effective mechanism for disturbance rejection and the transcortical (long-loop) stretch reflex is neglected due 


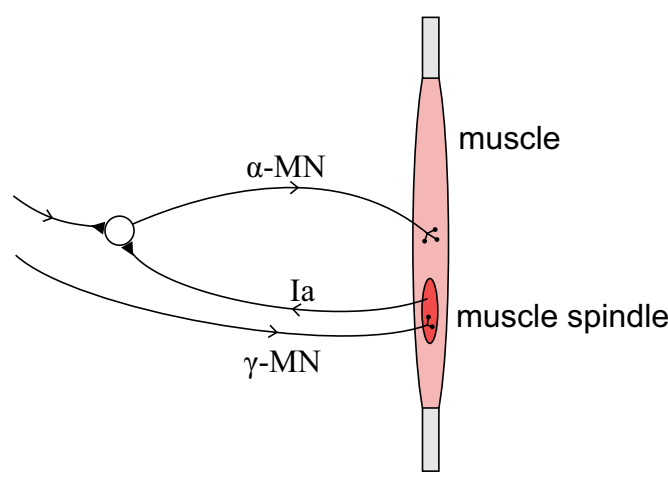

(a)

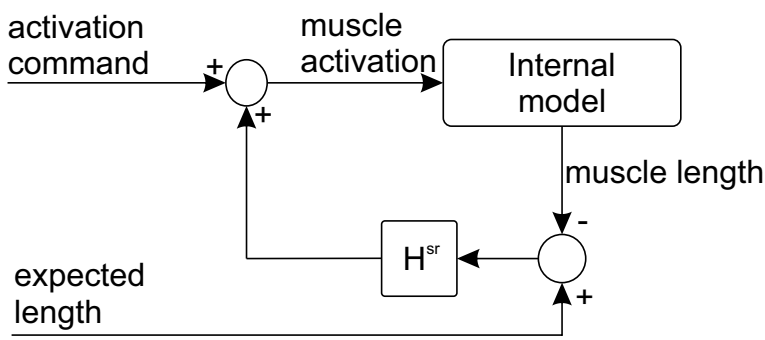

(b)

Figure 13. Stretch reflex mechanism in individual muscles (a) The schematic of the stretch reflex loop in humans (b) The mathematical realization of the stretch reflex in the arm model

to its complexity. Figure 13 presents a schematic of the stretch reflex mechanism in a human, and its block diagram representation in our model.

The accuracy of the stretch reflex mechanism depends on the muscle spindle model accuracy. The spindle model can either include highly detailed models of nuclear bag, bag fiber and chain fiber and afferent firing models [51-53], or a reduced-order and simplified model of spindle [54]. In this research, a reduced order model is used to simulate the response of the muscle spindles to a change in the muscle length. In this model, the muscle spindle response is considered as a nonlinear summation of the muscle length and muscle contraction velocity with a short time delay [55], as shown in the following transfer function:

$$
H^{s r}(S)=\frac{a_{\text {muscle }}(S)}{\Delta L_{\text {muscle }}(S)}=\left(K_{P}^{s r}+K_{D}^{s r} S\right) e^{-\tau_{d}^{s r} S}
$$

where $\Delta L_{\text {muscle }}$ and $a_{\text {muscle }}$ are individual muscle length error and activation, and $K_{P}^{s r}$, $K_{D}^{s r}$ and $\tau_{d}^{s r}$ are the proportional and derivative coefficients and the delay associated with short-loop stretch reflex mechanism, respectively.

The expected muscle length is found from the response of the internal model to the predicted optimal muscle activations (within the FD controller, see Figure 3). For each muscle, the error between the expected length and the actual length is calculated, and then multiplied by $H^{s r}$ to find the amount of corrective activation. It is then added to the original activation command to drive the muscle (see Figure 13(b)). 


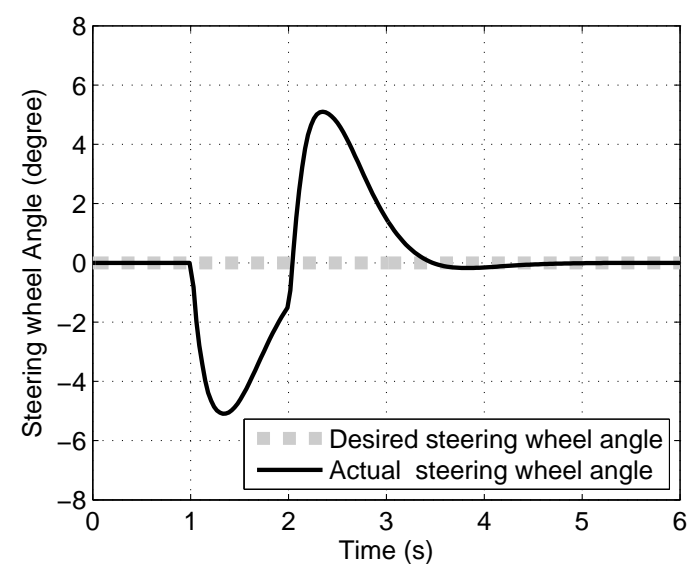

(a)

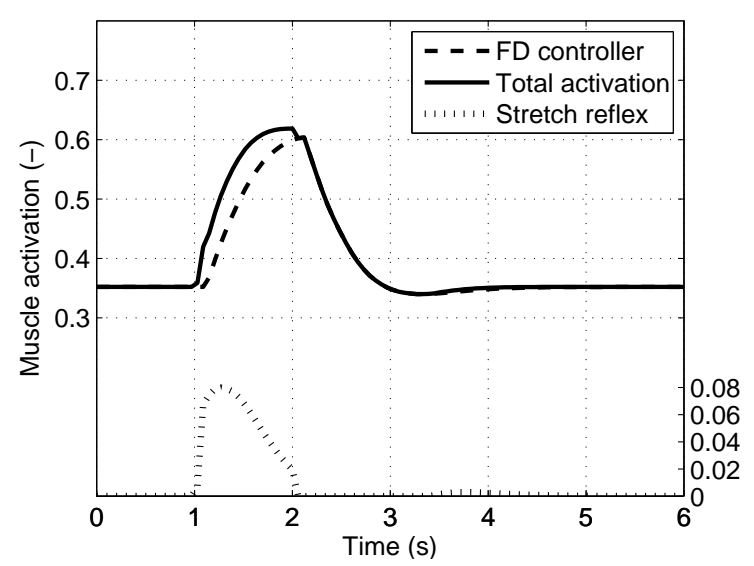

(b)

Figure 14. Disturbance rejection properties of the stretch reflex (a) Actual and desired steering wheel angle (b) The middle part of deltoid muscle force. The three graphs are the force distribution controller prediction, the stretch reflex addition and the total muscle force.

Figure 14 shows the disturbance rejection capabilities of the driver's reflex loop while steering with right hand only. Figure 14(a) shows that the driver tries to keep the steering wheel stationary, when the disturbance torque as described in section 3.2 .2 (see Figure 12(b)) is suddenly applied to the steering wheel. Figure 14(b) shows the middle portion of deltoid muscle response to the disturbance to stabilize the steering wheel. It can be seen that the stretch reflex responds quickly when the disturbance occurs but it takes more time for voluntary contribution of the CNS to notice, identify, and resist the disturbance.

\subsubsection{Intrinsic Stiffness}

Joint stiffness (impedance) modulation is another strategy employed by the CNS to resist external disturbances. This strategy works against all sorts of perturbations, but it is highly energy consuming. This strategy is voluntary and is a result of the physical structure and properties of muscle during contraction.

In this article, the equilibrium-point hypothesis [56] has been used to regulate the intrinsic properties of joints. Feldman proposed that the net passive moment at the joint is a function of joint angle and the equilibrium point, and the CNS manipulates the equilibrium point by adjusting the antagonistic co-contraction [56]. Here, the equilibrium point or expected arm kinematics is found from the response of internal model to the muscle activations as shown in Fig. 3 . 


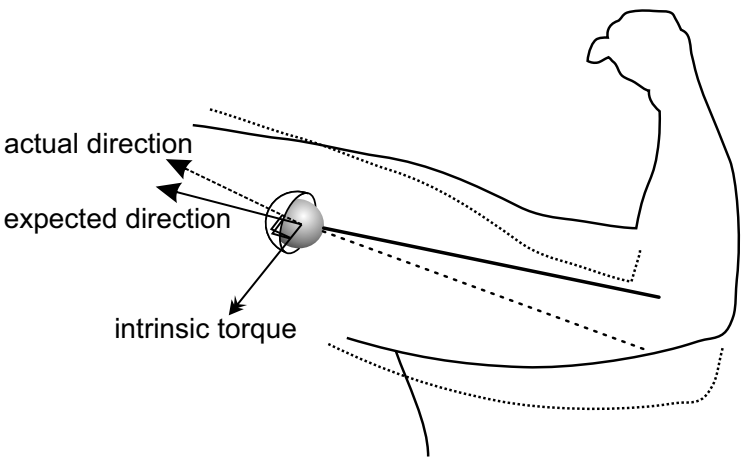

(a)

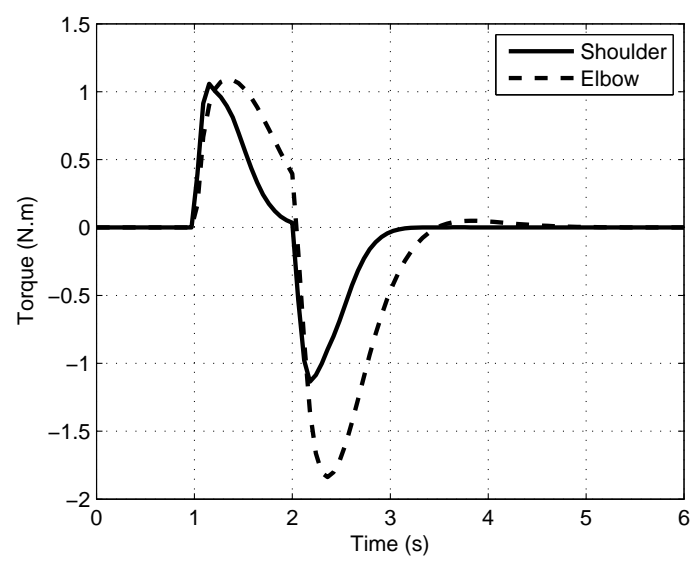

(b)

Figure 15. (a) Sketch of the joint stiffness mechanism (b) The passive joint moment produced by intrinsic properties of the muscles (reference frame as shown in Figure 2.1).

Since in the implementation, the SE element of Hill-type muscle model is not included, a moment is added to the joints to represent the intrinsic properties of the muscles wrapping the joint. This moment is the function of the deviation of the actual 3D direction of shoulder/elbow from its expected value and the muscle co-contraction ratio as illustrated in Eq. (6),

$$
\bar{T}_{j}^{I S}=\alpha\left(K_{j}^{I S} \theta_{j}+C_{j}^{I S} \dot{\theta}_{j}\right) \bar{n}
$$

where $\alpha$ is the muscle co-contraction ratio and $\theta_{j}$ and $\dot{\theta}_{j}$ are angle and angular velocity difference between the expected and actual shoulder/elbow directions. The passive moment is in the direction $\bar{n}$ which is the unit vector normal to the error plane, and is constructed using the cross product of the actual and expected direction vectors as shown in Fig. 15(a).

Figure 15(b) shows the passive joint moments produced by intrinsic properties of muscle when the muscle co-contraction ratio is assumed to be $30 \%$, in the scenario described in 3.2 .2 .

\section{Simulation Results and Discussion}

All the simulations are performed in the Matlab/Simulink environment. The models are exported to Matlab as an optimized C-code using the Maple CodeGeneration toolbox, 


\begin{tabular}{llll}
\hline Parameters & Description & Value & Unit \\
\hline$K_{s w}, C_{s w}$ & $\begin{array}{l}\text { identified steering stiffness and damping coeffi- } \\
\text { cients of the internal model }\end{array}$ & $7.5,0.9$ & {$[\mathrm{~N} / \mathrm{rad}],[\mathrm{N} / \mathrm{rad} \mathrm{s}]$} \\
$\alpha$ & $\begin{array}{l}\text { muscle co-contraction ratio (relaxed, tensed) } \\
\text { proportional and integral coefficients of distur- }\end{array}$ & $0 \%, 30 \%$ & {$[-]$} \\
$K_{P}^{e}, K_{I}^{e}$ & $\begin{array}{l}\text { bance observer } \\
\text { disturbance observer time delay (relaxed, }\end{array}$ & $\infty, 300$ & {$[\mathrm{rad}]$} \\
$\tau_{d}^{e}$ & $\begin{array}{l}\text { tensed) } \\
\text { proportional and integral coefficients of stretch }\end{array}$ & 9,1 & {$[\mathrm{~mm}],[\mathrm{mm} / \mathrm{s}]$} \\
$K_{P}^{s r}, K_{D}^{s r}$ & $\begin{array}{l}\text { reflex } \\
\tau_{d}^{s r}\end{array}$ & 25 & {$[\mathrm{~ms}]$} \\
$K_{s h}^{I S}, C_{s h}^{I S}$ & $\begin{array}{l}\text { intretch reflex time delay } \\
\text { inoulder stiffness and damping coefficients of }\end{array}$ & 12,8 & {$[\mathrm{rad}],[\mathrm{rad} / \mathrm{s}]$} \\
$K_{e}^{I S}, C_{e}^{I S}$ & $\begin{array}{l}\text { intrinsic stiffness and damping coefficients of el- } \\
\text { bow }\end{array}$ & 50,10 & {$[\mathrm{rad}],[\mathrm{rad} / \mathrm{s}]$} \\
\hline
\end{tabular}

Table 1. List of parameters used in the simulations

and the motor control framework is constructed in the Simulink environment. The parameters used in the simulations are summarized in Table 1. To secure the interpretation of results, our neuromusculoskeletal driver model has been verified against the experimentally validated neuromuscular driver model from [43]. In this comparison, the behavior of our driver model with relaxed and co-contracted muscles has been compared to the neuromuscular driver model in the presence of an external disturbance. This comparison showed a good correlation between the neuromuscular driver model and our driver model in terms of predicting the steering wheel angle [35]).

The simulation results for a step-like lane change maneuver steered with the right hand only at the speed of $10 \mathrm{~m} / \mathrm{s}$ are presented in Figures 16 and 17. Three simulations have been performed to show the full extent of the physics-based driver model capabilities. The first simulation is performed in the normal driving condition (no disturbance). The other two simulations correspond to driving with relaxed and tensed muscle conditions, when a two second long pulse-shaped disturbance occurs at the steering wheel. The torque disturbance at the steering wheel can be considered to be the result of a pothole or a strong cross wind.

In the relaxed condition, the voluntary contribution of the CNS (the FD controller) in disturbance rejection is neglected; in other words, it takes longer for the observer to estimate the altered states than the duration of disturbance itself. Additionally, the muscle co-contraction ratio is assumed to be zero; therefore, there is no intrinsic muscle stiffness properties in the relaxed driving condition. In the tensed driving condition, the driver actively tries to identify and reject the disturbance. In this case, the latency 


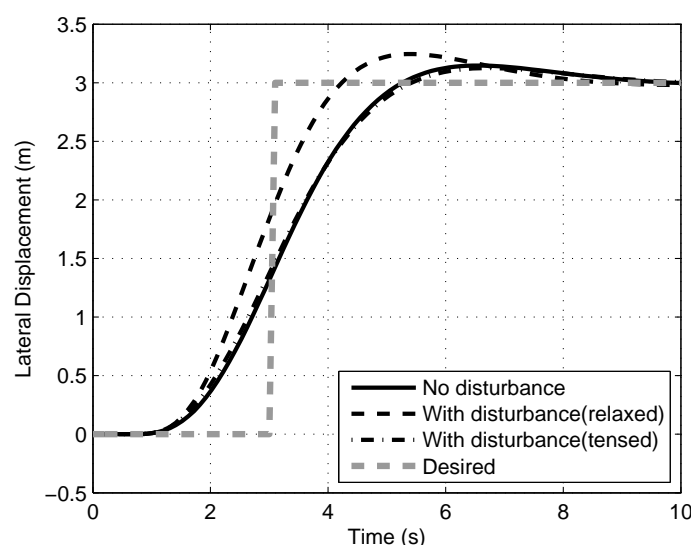

(a)

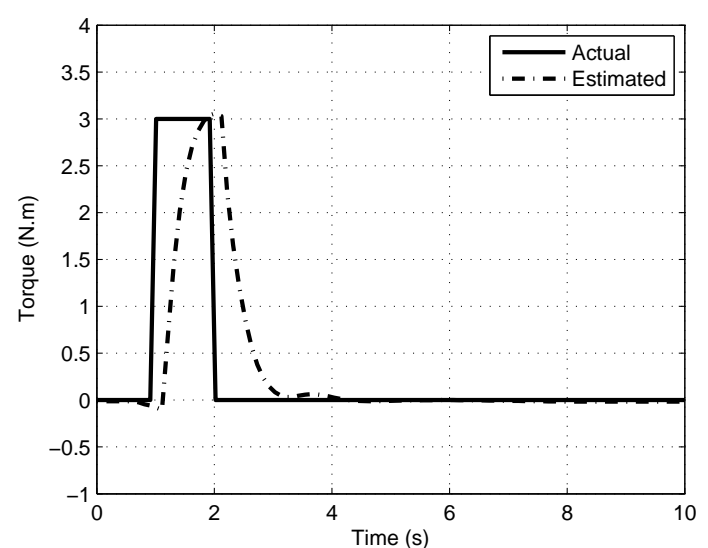

(b)

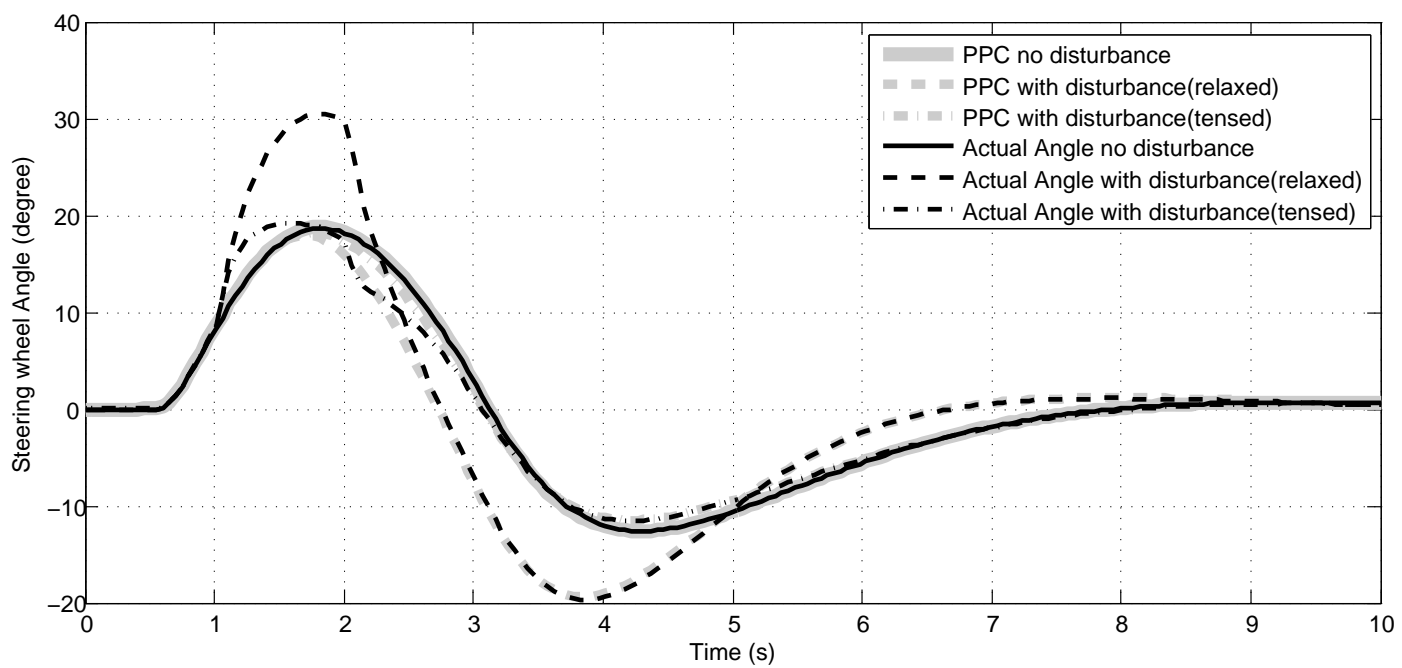

(c)

Figure 16. Simulation results of the lane changing maneuver (a) Desired and actual lateral displacement with and without disturbance (b) Actual and estimated disturbance torque applied to the steering wheel (c) Desired and actual steering wheel angles, with and without disturbance. PPC is the output of path planning controller.

associated with the disturbance observer and the muscle co-contraction ratio are assumed to be $300 \mathrm{~ms}$ and $30 \%$, respectively.

Figures 16 and 17 show the effect of the external disturbance on all layers of the motor control framework. Figure 16(a) shows the comparison between the lateral displacement of the vehicle in the presence and absence of the disturbance. It can be seen that, for all three conditions, the driver starts to steer 2.5 seconds before the desired trajectory, which is consistent with predictive ability of the path planning controller; the responses also settle down at about $\mathrm{t}=9 \mathrm{~s}$. However, the deviation from the desired path (overshoot) of the perturbed vehicle is different across the three simulations. In the relaxed driving 
condition, the overshoot is more than the tensed condition and unperturbed vehicle. This difference is a result of the difference in the actual steering wheel angles in Figure 16(c).

As a result of the disturbance, the reflex addition to the muscle forces is substantial. However, despite the stretch reflex corrections, the arm cannot follow the desired steering wheel trajectory. This deviation in the steering wheel angle, in turn, results in a deviation in the vehicle's path. The path planning controller then corrects the path by providing a new desired steering wheel angle, which finally results in different muscle activations that are predicted by the force distribution controller compared to the case with no external disturbance.

In addition to the disturbance itself, Figure 16(b) also shows the performance of the disturbance observer for the tensed driving condition. It can be seen that the observer does not start to identify the disturbance at the steering wheel until $300 \mathrm{~ms}$ (the observer time delay) after its onset, after which it quickly reaches the actual disturbance torque. The time delay associated with the observer is the latency regarding the information transmission to and processing in the sensorimotor area of brain, which leads to voluntary response of the driver to overcome the disturbances. The slight difference between the estimated and actual disturbance in the no disturbance zone is due to the difference between the internal model and the actual vehicle model.

At the beginning and end of the disturbance we have the largest error in the disturbance estimation, which causes the largest error in the actual and desired steering wheel angles. These periods are the times before the voluntary action intervenes and reduces the disturbance. At the same time, because of this error in the steering wheel angle, the first layer of the motor control framework, the path-following controller, intervenes and corrects the desired steering wheel angle to follow the new desired path.

Figure 17 shows the required muscle activations wrapping the shoulder to perform the expected steering wheel angle in the three simulation conditions. It can be seen that there is no antagonist muscle activation in the no disturbance condition as shown in Fig. 17(a), as the gravity helps the driver to turn the steering wheel clockwise. In this condition, the anterior and the middle part of deltoid muscle (DELT1, DELT2), coracobrachialis muscle (CORB), infraspinatus (INFRA) and pectoralis major (PECT) are responsible for the steering action. On the other hand, in the relaxed condition, as a result of the disturbance and consequently the deviation of steering angle from its expected value, the antagonist muscles are activated to first stabilize the steering wheel and then to return it 
to its expected value. In the tensed driving condition, the muscle activations are already built up to stabilize the steering if there is a disturbance. As shown in Fig. 17(c), both agonist and antagonist muscles are activated to reject the disturbance; thus the vehicle closely follows the path of the unperturbed vehicle.

\section{Conclusions and Future work}

Having a clear understanding of the dynamical system is crucial in control system design, it provides knowledge of the system that can be used to reduce development time and cost. In this research, a high-fidelity vehicle model and a neuromusculoskeletal driver model are developed in silico to replicate the interaction between the driver and vehicle. A hierarchical approach is used to capture the complexity of neuromuscular dynamics and the central nervous system in the coordination of the driver upper limb activity, with a particular attention to steering disturbance rejection.

This physics-based driver model can be used to study the underlying dynamics of a human driver in stabilizing the vehicle in the presence of external disturbances, or when a steering fault occurs in the steering system. This model is also equipped with the driver's internal representation of the vehicle, musculoskeletal disturbance observer, and muscle co-contraction ratio, which can represent the driver's steering skill and simulate relaxed or tensed driving conditions. The model can be used to quantify objective criteria such as fatigue and muscle stress for drivers of different age, gender, and physical ability, which can be used to support the development of new assistive steering technologies.

\section{Acknowledgement}

The authors would like to thank the Ontario Centres of Excellence (OCE), the Natural Sciences and Engineering Research Council of Canada (NSERC), Toyota, and Maplesoft for their support of this research.

\section{References}

[1] N. Mehrabi and J. McPhee, "Steering feel improvement for different driver types using model-based control," in Proceedings of the ASME 2014 International Design Engineering Technical Conferences, (Buffelo, New York), 2014. 
[2] C. MacAdam, "Understanding and Modeling the Human Driver," Vehicle System Dynamics, vol. 40, no. 1-3, pp. 101-134, 2003.

[3] R. S. Sharp and H. Peng, "Vehicle dynamics applications of optimal control theory," Vehicle System Dynamics, vol. 49, no. 7, pp. 1073-1111, 2011.

[4] C. MacAdam, "Application of an optimal preview control for simulation of closed-loop automobile driving," IEEE Transactions on Systems, Man and Cybernetics, vol. 11, no. 6, pp. 393-399, 1981.

[5] K. Jalali, S. Lambert, and J. McPhee, "Development of a path-following and a speed control driver model for an electric vehicle," SAE Technical Paper 2012-01-0250, 2012.

[6] R. Sharp and V. Valtetsiotis, "Optimal preview car steering control," Selected papers from 20th International Congress of Theoretical and Applied Mechanics, Supplement to Vehicle System Dynamics, vol. 35, pp. 101-117, 2001.

[7] D. Cole, A. Pick, and A. Odhams, "Predictive and linear quadratic methods for potential application to modeling driver steering control," Vehicle System Dynamics: International Journal of Vehicle Mechanics and Mobility, vol. 44, no. 3, pp. 259-284, 2006.

[8] D. Weir and D. McRuer, "Dynamics of Driver Vehicle Steering Control," Automatica, vol. 6, no. 1, pp. 87-98, 1970.

[9] N. Mehrabi, R. Sharif Razavian, and J. McPhee, "A three-dimentional musculoskeletal driver model to study steering tasks," in International Design Engineering Technical Conference 85 Computers and Information in Engineering Conferences, (Portland, Oregon), 2013.

[10] C. Droogendijk, A new neuromuscular driver model for steering system development. Master thesis, Delft University of Technology, Delft, Netherlands, 2010.

[11] C. Sentouh and P. Chevrel, "A human-centred approach of steering control modelling," in Proceedings of the 21st IAVSD Symposium on Dynamics of Vehicles on Roads and Tracks, (Stockholm, Sweden), 2009.

[12] D. Katzourakis, C. Droogendijk, D. Abbink, R. Happee, and E. Holweg, "Driver model with visual and neuromuscular feedback for objective assessment of automotive steering systems," in International Symposium on Advanced Vehicle Control (AVEC), (Loughborough, UK), 2010.

[13] A. Pick and D. Cole, "A mathematical model of driver steering control including neuromuscular dynamics," Journal of Dynamic Systems, Measurement, and Control, vol. 130, no. 3, p. 031004, 2008.

[14] A. Pick and D. Cole, "Neuromuscular dynamics and the vehicle steering task," in The 18th International Association for Vehicle System Dynamics Symposium, (Kanagawa, Japan), October 2003.

[15] A. Pick and D. Cole, "Neuromuscular dynamics in the driver-vehicle system," Vehicle System Dynamics: International Journal of Vehicle Mechanics and Mobility, vol. 44, no. sup1, pp. 624-631, 2006 .

[16] D. Cole, "Neuromuscular dynamics and steering feel," in Proceedings of SteeringTech, (TU Munich, Germany), 2008.

[17] N. Mehrabi, R. Sharif Razavian, and J. McPhee, "A Physics-Based Musculoskeletal Driver Model to Study Steering Tasks, doi: 10.1115/1.4027333," Journal of Computational and Nonlinear Dynamics, 
vol. 10, no. 2, 2015 .

[18] R. Maas and S. Leyendecker, "Optimal control of biomechanical motion using physiologically motivated cost functions," in The 2nd Joint International Conference on Multibody System Dynamics, vol. 12, (Stuttgart), pp. 99-100, 2012.

[19] R. Shadmehr and S. Wise, The computational neurobiology of reaching and pointing: a foundation for motor learning. MIT Press, 2005.

[20] F. van der Helm, A. Schouten, E. de Vlugt, and G. Brouwn, "Identification of intrinsic and reflexive components of human arm dynamics during postural control," Journal of Neuroscience Methods, vol. 119 , no. 1 , pp. 1-14, 2002 .

[21] A. V. Hill, "The heat of shortening and the dynamic constants of muscle," Proceedings of the Royal Society of London. Series B, Biological Sciences, vol. 126, no. 843, pp. 136-195, 1938.

[22] D. G. Thelen, F. C. Anderson, and S. L. Delp, "Generating dynamic simulations of movement using computed muscle control," Journal of Biomechanics, vol. 36, no. 3, pp. 321-328, 2003.

[23] D. R. WILKIE, "The mechanical properties of muscle.," British medical bulletin, vol. 12, pp. 177182, 1956.

[24] F. E. Zajac, "Muscle and tendon: properties, models, scaling, and application to biomechanics and motor control.," Critical reviews in biomedical engineering, vol. 17, pp. 359-411, 1989.

[25] S. Heitmann, N. Ferns, and M. Breakspear, "Muscle co-contraction modulates damping and joint stability in a three-link biomechanical limb," Frontiers in neurorobotics, vol. 5, 2011.

[26] J. Winters, L. Stark, and a. H. Seif-Naraghi, "An analysis of the sources of musculoskeletal system impedance," Journal of Biomechanics, vol. 21, no. 12, pp. 1011-25, 1988.

[27] D. J. Cole, "Steering Feedback Mathematical Simulation of Effects on Driver and Vehicle," $A T Z a-$ utotechnology, vol. 8, 2008.

[28] W. Hoult and D. J. Cole, "A neuromuscular model featuring co-activation for use in driver simulation," Vehicle System Dynamics, vol. 46, no. sup1, pp. 175-189, 2008.

[29] N. Mehrabi, M. Sharif, and J. McPhee, "Study of human steering tasks using a neuromuscular driver model," in Advanced Vehicle and Control Conference (AVEC), (Seoul, Korea), 2012.

[30] R. W. Bassett, A. O. Browne, B. F. Morrey, and K. N. An, "Glenohumeral muscle force and moment mechanics in a position of shoulder instability," Journal of Biomechanics, vol. 23, pp. 405-415, 1990.

[31] S.-B. Lee and K.-N. An, "Dynamic glenohumeral stability provided by three heads of the deltoid muscle.," Clinical orthopaedics and related research, pp. 40-47, 2002.

[32] A. Huxley, "Muscle Contraction and theories of Contraction," Progress in Biophysics and Biophysical Chemistry, vol. 7, pp. 255-318, 1957.

[33] N. Mehrabi and J. McPhee, "Evaluation of a Musculoskeletal Arm Model for Automobile Drivers using Electromyography," in 10th Conference of the International Shoulder Group, (Waterloo, Ontario), 2014.

[34] N. Mehrabi and J. McPhee, "Evaluation of a musculoskeletal arm model for automobile drivers using electromyography," in 7th World Congress of Biomechanics, (Boston, Massachusetts), 2014.

[35] N. Mehrabi, Dynamics and Model-Based Control of Electric Power Steering Systems. PhD thesis, 
University of Waterloo, Waterloo. Ontario, 2014.

[36] A. Hall, T. Uchida, F. Loh, C. Schmitke, and J. McPhee, "Reduction of a Vehicle Multibody Dynamic Model Using Homotopy Optimization," Archive of Mechanical Engineering, vol. 60, no. 1, pp. 23-35, 2013.

[37] A. Hall and J. McPhee, "Automation of Adams/Car K\&C Correlation using MATLAB," SAE Technical Paper 2014-01-0847, 2014.

[38] D. Cole, A. Pick, and A. Odhams, "Predictive and linear quadratic methods for potential application to modelling driver steering control," Vehicle System Dynamics, vol. 44, no. 3, pp. 259-284, 2006.

[39] N. Kim and D. J. Cole, "A model of driver steering control incorporating the driver's sensing of steering torque," Vehicle System Dynamics, vol. 49, no. 10, pp. 1575-1596, 2011.

[40] MathWorks, "Model Predictive Control Toolbox," URL: http://www.mathworks.com/products/mpc/, 2014.

[41] R. D. Crowninshield and R. A. Brand, "A physiologically based criterion of muscle force prediction in locomotion," Journal of Biomechanics, vol. 14, no. 11, pp. 793-801, 1981.

[42] R. Happee, "Inverse dynamic optimization including muscular dynamics, a new simulation method applied to goal directed movements," Journal of Biomechanics, vol. 27, no. 1, pp. 953-960, 1994.

[43] A. Pick and D. Cole, "Dynamic properties of a driver's arms holding a steering wheel," Proceedings of the Institution of Mechanical Engineers, Part D: Journal of Automobile Engineering, vol. 12, no. 221, pp. 1475-1486, 2007.

[44] R. Deborne, A. Barthou, D. Toffin, G. Reymond, and A. Kemeny, "Simulation study of driver stress and performance to an unexpected steering critical event," in Proceedings of the Driving Simulation Conference, 2008.

[45] A. J. Pick and D. J. Cole, "Driver steering and muscle activity during a lane-change manoeuvre," Vehicle system dynamics, vol. 45, no. 9, pp. 781-805, 2007.

[46] E. Forster, U. Simon, P. Augat, and L. Claes, "Extension of a state-of-the-art optimization criterion to predict co-contraction.," Journal of biomechanics, vol. 37, no. 4, pp. 577-81, 2004.

[47] F. Anderson and M. Pandy, "Dynamic Optimization of Human Walking," Journal of Biomechanical Engineering, vol. 123, no. 5, p. 381, 2001.

[48] M. Sharif Shourijeh and J. McPhee, "Optimal Control and Forward Dynamics of Human Periodic Motions Using Fourier Series for Muscle Excitation Patterns," Journal of Computational and Nonlinear Dynamics, vol. 9, no. 2, p. 021005, 2013.

[49] U. Ackermann, PDQ: Physiology, vol. 95. McGraw-Hill Europe, 2002.

[50] L. Ting, K. van Antwerp, J. Scrivens, L. McKay, T. Welch, J. Bingham, and S. DeWeerth, "Neuromechanical tuning of nonlinear postural control dynamics," Chaos (Woodbury, N.Y.), vol. 19, no. 2, p. 26111, 2009.

[51] Z. Hasan, "A model of spindle afferent response to muscle stretch," Journal of Neurophysiology, vol. 49, no. 4, pp. 989-1006, 1983.

[52] M. Mileusnic, I. Brown, N. Lan, and G. Loeb, "Mathematical models of proprioceptors. I. Control and transduction in the muscle spindle.," Journal of Neurophysiology, vol. 96, no. 4, pp. 1772-88, 
2006.

[53] C. Gielen and J. Houk, "A model of the motor servo: incorporating nonlinear spindle receptor and muscle mechanical properties," Biological Cybernetics, vol. 57, no. 4-5, pp. 217-231, 1987.

[54] N. Bhushan and R. Shadmehr, "Computational nature of human adaptive control during learning of reaching movements in force fields.," Biological Cybernetics, vol. 1, no. 81, pp. 39-60, 1999.

[55] E. Burdet, D. Franklin, and T. Milner, Human Robotics. The MIT Press, 2013.

[56] A. Feldman and M. Levin, "The origin and use of positional frames of reference in motor control," Behavioral and Brain Sciences, vol. 18, pp. 723-806, 1995.

[57] M. Sharif Shourijeh, Optimal Control and Multibody Dynamic Modelling of Human Musculoskeletal Systems. PhD thesis, University of Waterloo, Waterloo, Ontario, 2013.

[58] E. Pennestri, R. Stefanelli, P. Valentini, and L. Vita, "Virtual musculo-skeletal model for the biomechanical analysis of the upper limb," Journal of Biomechanics, vol. 40, no. 6, pp. 1350-61, 2007.

[59] R. Gopura, K. Kiguchi, and E. Horikawa, "A study on human upper-limb muscles activities during daily upper-limb motions," International Journal of Bioelectromagnetism, vol. 12, no. 2, pp. 54-61, 2010.

[60] G. Yamaguchi, A. Sawa, D. Moran, M. Fessler, and J. Winters, Multiple Muscle Systems, Biomechanics and Movement Organization. Springer-Verlag, 1990. 


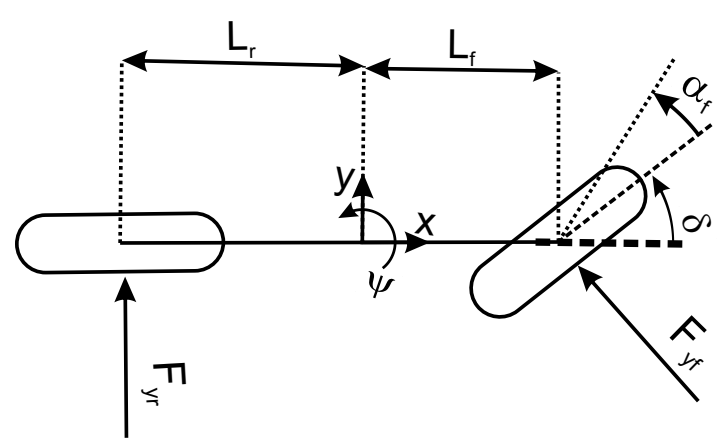

Figure A1. Schematic view of the linear bicycle model.

\section{Appendix A. Bicycle Model}

A linear bicycle model of the vehicle as shown in Fig. A1 is confined in the model predictive controller of the motor control framework. This vehicle model is represented in the following linear state space representation,

$$
\dot{\mathbf{x}}=\mathbf{A} \mathbf{x}+\mathbf{B} u
$$

where $x$ are the state variables and $u$ is the steering wheel angle. The matrices and vectors in Eq. (A1) are written as,

$$
\mathbf{A}=\left[\begin{array}{cccc}
-\frac{2 C_{f}+2 C_{r}}{M U_{0}} & -\left(U+\frac{2 l_{f} C_{f}-2 l_{r} C_{r}}{M U_{0}}\right) & 0 & 0 \\
-\frac{2 l_{f} C_{f}-2 l_{r} C_{r}}{I_{z} U} & -\frac{2 l_{f}^{2} C_{f}-2 l_{r}^{2} C_{r}}{I_{z} U} & 0 & 0 \\
1 & 0 & 0 & U_{0} \\
0 & 1 & 0 & 0
\end{array}\right] \mathbf{B}=\left[\begin{array}{c}
\frac{2 C_{f}}{M G_{r}} \\
\frac{2 l_{f} C_{f}}{I_{z} G_{r}} \\
0 \\
0
\end{array}\right]
$$

and $\mathbf{x}=\left[\begin{array}{llll}x_{1} & x_{2} & x_{3} & x_{4}\end{array}\right]^{T}$, where $x_{3}, x_{1}$ are the vehicle lateral position and velocity and $x_{4}, x_{2}$ are the vehicle yaw angle and rate, respectively. The bicycle model parameters and descriptions are shown in table A1.

\begin{tabular}{llcc}
\hline Parameters & Description & Value & Unit \\
\hline$C_{f}, C_{r}$ & front and rear tire cornering stiffness & 117000 & {$[\mathrm{~N} / \mathrm{rad}]$} \\
$U_{0}$ & longitudinal vehicle velocity & 10 & {$[\mathrm{~m} / \mathrm{s}]$} \\
$M$ & vehicle mass & 2077 & {$[\mathrm{~kg}]$} \\
$I_{z}$ & vehicle yaw inertia & 1995.78 & {$\left[\mathrm{~kg} \mathrm{~m} \mathrm{~m}^{2}\right]$} \\
$l_{f}, l_{r}$ & distance to the front and rear axles from CG & $1.192,1.548$ & {$[\mathrm{~m}]$} \\
$G_{r}$ & steering gear ratio & 15.29 & {$[-]$} \\
\hline
\end{tabular}

Table A1. List of identified bicycle model parameters used in the path planning controller 


\section{Appendix B. Hill-type Muscle Model}

The model used to simulate the muscle dynamics is inspired from the popular Hill muscle model $[21,22]$. As shown in Fig. B1, the Hill muscle model consists of a Contractile Element (CE) and a Parallel Elastic element (PE) in series with a Series Elastic element (SE). In this article, the tendon dynamics (SE) are neglected to simplify the mathematical representation of muscle dynamics. Therefore, the muscle model is reduced to the $\mathrm{CE}$ element in parallel with the PE element. Based on these assumptions, the muscle force can be found as follows:

$$
F_{T M}(t)=F_{0}^{\max }\left\{F_{P E}\left(t, L_{M}\right)+F_{C E}\left(t, a, L_{M}, V_{M}\right)\right\} \cos \left(\alpha_{p}\right)
$$

where $F_{P E}$ and $F_{C E}$ are the passive and active forces of the muscle, respectively. $L_{M}, V_{M}$, $\alpha_{p}$ and $F_{0}^{\max }$ are the muscle length, contraction velocity, pennation angle and maximum isometric muscle force, respectively. The muscle activation $(a)$ represents the fraction of active motor units in the muscle (between 0 and 1), and since the SE element is removed, the pennation angle for all muscles is assumed to be zero.

The force generated by the active part of muscle (CE) can be separated into forcelength and force-velocity relations [22], which is scaled by the activation level of the muscle as shown in Eq. (B2). A schematic diagram of these two relations is shown in Fig. B2.

$$
F_{C E}\left(t, a, V_{M}\right)=a(t) F_{C E}^{L}\left(t, L_{M}\right) F_{C E}^{V}\left(t, a, L_{M}, V_{M}\right)
$$

The force-length relation is described by the normal distribution function as follows [22]:

$$
F_{C E}^{L}\left(t, L_{M}\right)=\mathrm{e}^{-\left(\frac{L_{M}(t)}{L_{M}^{o t}}-1\right)^{2} / \gamma}
$$

where the shaping factor $\gamma$ is set to 0.45 , and the length of muscle in the initial posture is selected as the optimal length of muscle $\left(L_{M}^{o p t}\right)$. The driver is holding the steering wheel 


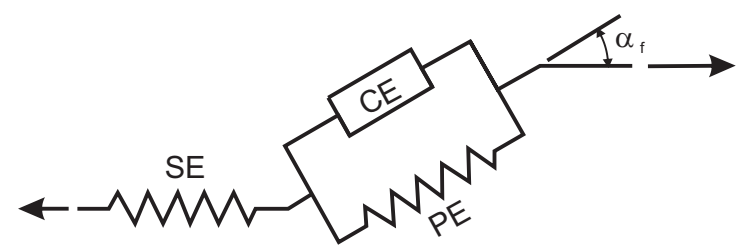

Figure B1. An example of a Hill-type muscle model [22]. The Hill muscle model consists of series elastic element (SE), parallel elastic element (PE) and contractile element (CE) as the active part of muscle.

at 3 o'clock position in his/her initial posture, and the steering axis is parallel to the line connecting the shoulder to the steering wheel. The force-contraction velocity dependent relation is approximated by the following formula $[22,57]$ :

$$
F_{C E}^{V}= \begin{cases}\frac{V_{M} / V_{M}^{\max } L_{M}^{o p t}+A V_{M}^{\max }}{V_{M} / V_{M}^{\max } L_{M}^{o p t} A_{f}+A V_{M}^{\max }} & V_{M}<0 \\ \frac{V_{M} B \bar{F}_{\max }^{\text {len }} / V_{M}^{\max } L_{M}^{o p t}+A C V_{M}^{\max }}{V_{M} B / V_{M}^{\max } L_{M}^{o p t} A_{f}+A C V_{M}^{\max }} & V_{M}>0\end{cases}
$$

where $V_{M}^{\max }$, the maximum contraction velocity of the muscle, is set to $10\left(L_{M}^{o p t} / s\right)$ and $\mathrm{A}, \mathrm{B}$ and $\mathrm{C}$ are shape factors calculated by $A=0.25+0.75 a(t), B=2+2 / A_{f}$ and $C=\bar{F}_{l e n}^{\max }-1$, respectively. $\bar{F}_{l e n}^{\max }$, the maximum normalized muscle force achievable during lengthening, is set to 1.4 and $A_{f}$, a force-velocity shape factor, is set to 0.25 .

The force-length relationship of the PE element of the muscle as shown in Fig. 2(b) is represented by an exponential function [22]:

$$
F_{P E}\left(t, L_{M}\right)=\frac{\mathrm{e}^{k_{p e}\left(\frac{L_{M}(t)}{L_{M}^{p t}}-1\right)} / \epsilon_{0}^{m}-1}{\mathrm{e}^{k_{p e}}-1}
$$

where $k_{p e}$, a shape factor, is set to 5 , and $\epsilon_{0}^{m}$, the passive muscle strain due to the maximum isometric muscle force, is set to 0.6. 


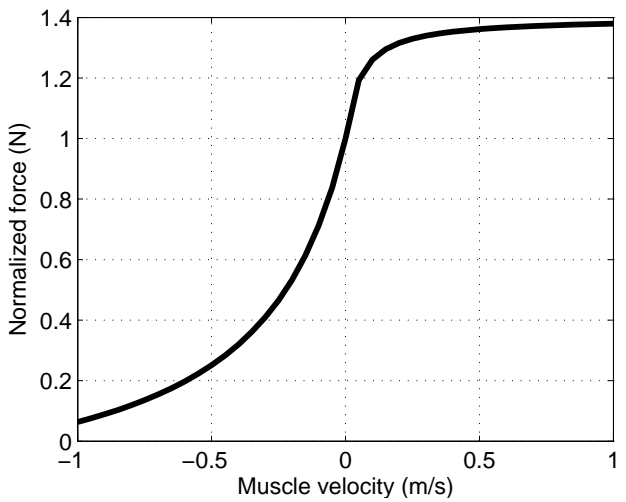

(a)

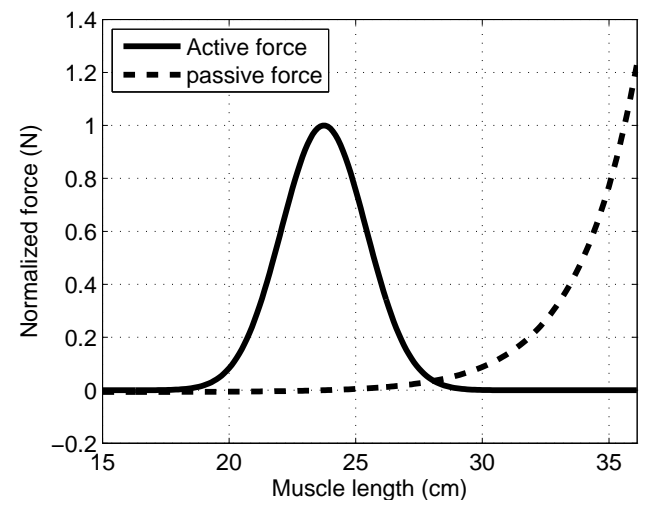

(b)

Figure B2. (a) The muscle force-velocity dependent relation $\left(F_{C E}^{V}\right)$, (b) The muscle force-length dependent relation of CE (active force) and PE element (passive force)

\section{Appendix C. Muscle Parameters}

The muscle path parameters used in the 3D musculoskeletal arm model are adopted from the published research articles [22, 58-60], and are summarized in Table C1. The muscle origin and insertion coordinates are given with respect to the local reference frames as shown in Figure 2.1.

\begin{tabular}{|c|c|c|c|c|c|c|c|c|c|c|}
\hline \multirow{2}{*}{ \# } & \multirow{2}{*}{ muscle } & \multirow{2}{*}{$\begin{array}{l}\max \\
\text { iso. } \\
\text { force }(\mathrm{N})\end{array}$} & \multirow{2}{*}{$\begin{array}{c}\text { first } \\
\text { connection } \\
\text { (origin) }^{* *}\end{array}$} & \multicolumn{3}{|c|}{ coordinate $(\mathrm{mm})$} & \multirow{2}{*}{$\begin{array}{c}\text { second } \\
\text { connection } \\
(\text { insertion })^{* *}\end{array}$} & \multicolumn{3}{|c|}{ coordinates $(\mathrm{mm})$} \\
\hline & & & & $\mathrm{x}$ & $\mathrm{y}$ & $\mathrm{z}$ & & $\mathrm{x}$ & $\mathrm{y}$ & $\mathrm{z}$ \\
\hline 1 & Coracobrachialis (CORB) & 63 & torso & 20 & 30 & 35 & humerus & 174 & 21 & 0 \\
\hline 2 & Deltoid (DELT1)* & 240 & torso & -30 & 40 & 15 & humerus & 106 & -24 & -11 \\
\hline 3 & Deltoid (DELT2)* & 200 & torso & -9 & 64 & 25 & humerus & 119 & -9 & 9 \\
\hline 4 & Deltoid (DELT3)* & 200 & torso & -45 & 35 & -10 & humerus & 95 & -9 & 9 \\
\hline 5 & Latissimus dorsi (LAT) & 360 & torso & -35 & 90 & -125 & humerus & 50 & 0 & -13 \\
\hline 6 & Pectoralis major (PECM) & 210 & torso & 45 & 95 & -125 & humerus & -14 & 1 & 2 \\
\hline 7 & Supraspinatus (SUPSP) & 98 & torso & -20 & 90 & 35 & humerus & -14 & 17 & 27 \\
\hline 8 & Biceps brachii (BICshort) & 47 & torso & 0 & 0 & 15 & ulna & 38 & 0 & 10 \\
\hline 9 & Biceps brachii (BIClong) & 90 & torso & 0 & 15 & 10 & ulna & 38 & 0 & 10 \\
\hline 10 & Infraspinatus (INFRA)* & 210 & torso & -15 & 80 & -40 & humerus & 28 & -19 & 27 \\
\hline 11 & Anconeus (ANC)* & 40 & humerus & 265 & 5 & -19 & humerus & 42 & 12 & -29 \\
\hline 12 & Triceps brachii (TRIlong)* & 135 & torso & -25 & 20 & -20 & radius & 38 & 27 & -20 \\
\hline 13 & Triceps brachii (TRImed)* & 108 & humerus & 78 & 11 & -10 & ulna & 38 & 27 & -20 \\
\hline 14 & Brachialis (BRA) & 167 & humerus & 176 & -8 & 16 & radius & 33 & 5 & 10 \\
\hline 15 & Brachioradialis (BRD) & 45 & humerus & 246 & -27 & 0 & radius & 283 & -12 & 0 \\
\hline
\end{tabular}

Table C1. List of muscle origin/insertion points used in the 3D arm model 